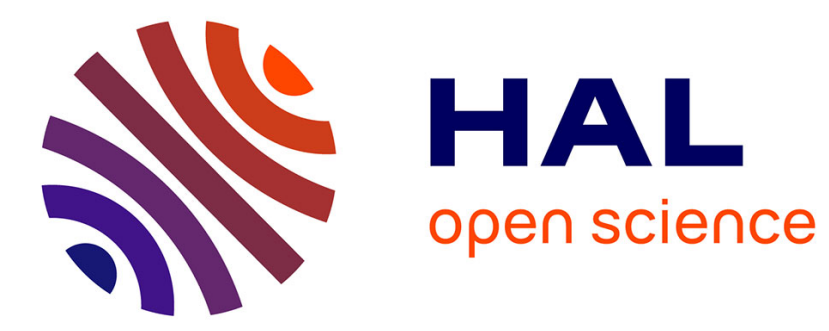

\title{
Digital I/Q Imbalance Correction for Full-Duplex Dual-Band OFDM Radio Transceivers I Introduction
}

Zhaowu Zhan, Guillaume Villemaud, Florin Hutu, Jean-Marie Gorce

\section{To cite this version:}

Zhaowu Zhan, Guillaume Villemaud, Florin Hutu, Jean-Marie Gorce. Digital I/Q Imbalance Correction for Full-Duplex Dual-Band OFDM Radio Transceivers I Introduction. International Journal of Microwave and Wireless Technologies, 2016, 8 (3), pp.505-519. 10.1017/S1759078715000811 . hal01244725

\section{HAL Id: hal-01244725 \\ https://hal.inria.fr/hal-01244725}

Submitted on 16 Dec 2015

HAL is a multi-disciplinary open access archive for the deposit and dissemination of scientific research documents, whether they are published or not. The documents may come from teaching and research institutions in France or abroad, or from public or private research centers.
L'archive ouverte pluridisciplinaire $\mathbf{H A L}$, est destinée au dépôt et à la diffusion de documents scientifiques de niveau recherche, publiés ou non, émanant des établissements d'enseignement et de recherche français ou étrangers, des laboratoires publics ou privés. 


\section{Digital I/Q Imbalance Correction for Full-Duplex Dual-Band OFDM Radio Transceivers}

Zhaowu Zhan, Guillaume Villemaud, Florin Hutu and Jean-Marie Gorce

Université de Lyon, INRIA, INSA-Lyon, CITI-INRIA, F-69621, Villeurbanne, France

This paper presents a Full-Duplex Dual-Band (FDDB) OFDM radio architecture that enables the radio transceiver to be more flexible and provides a viable radio link capacity gain. A simple but practical $I / Q$ imbalance estimation and compensation method, based on the frequency-flat-fading behavior of the self-interference channel, is proposed. The performance of the proposed $I / Q$ imbalance compensation method is evaluated by system level simulations conducted with ADS and Matlab. The co-simulation results show that the proposed radio transceiver could potentially increase the physical layer transmission rate by four times compared to the conventional radio link at the cost of tolerable loss of BER performance. The $I / Q$ imbalance compensation method can effectively compensate both high and low $I / Q$ imbalance without the problem of algorithm convergence.

Keywords: Authors should not add keywords, as these will be chosen during the submission process (see http://journals.cambridge.org/data/relatedlink/MRF_topics.pdf for the full list)

Corresponding author: Zhaowu Zhan; email: zhaowu.zhan@insa-lyon.fr; phone: +33650 242354

\section{Introduction}

The growing demand for various multimedia services and increasingly pervasive wireless radios call for the development of advanced and flexible radio transceivers to support high data rates and different wireless standards over limited radio resources. However, seeking a highly flexible and agile radio transceiver is always a big challenge. Fortunately, the FDDB radio's noticeable gain in rate and capability of processing two different types of signals on two separate spectrum fragments [1] promote it as a flexible radio front-end. Full-duplex is defined as a radio which can simultaneously transmit and receive in the same frequency band [2,3]. More globally, Full-duplex radio shows a great potential for future flexible radio systems. Nowadays, dual-band radios can simultaneously process two different signals, even the ones from distinct physical layers. This can be accomplished by using one common radio frequency $(\mathrm{RF})$ front-end with double I/Q structure [4]. FDDB radio, based on the combination of Full-Duplex and Dual-Band radio, enables the radio node to simultaneously transmit and receive on each separate spectrum channel with one common RF front-end. Such a capability could also be exploited to enhance the radio resource sharing and coexistence. Portable radio terminals, such as smartphones, have to 
accommodate a growing list of wireless standards such as WiFi, Bluetooth, 3G, 4G and even future $5 \mathrm{G}$. Current practical implementation is to use separate radios and antennas to meet the different standards requirements, which is expensive and puts high requirements on the space of portable consumer devices. Instead, FDDB could enable the radio terminal to accommodate two different wireless standards with one common $\mathrm{RF}$ front-end.

Furthermore, OFDM-based capabilities of sensing and spectrum shaping together with its flexibility make it probably the best transmission technology for flexible radios [5]. Therefore, FDDB OFDM radio transceivers based on the RF front-end proposed in [1] can be the flexible radio transceiver for the future wireless network.

Although the full-duplex enables the radio terminal to be more flexible, the big challenge behind is the strong self-interference that can saturate the radio receiver and severely penalizing the performance of the system. Besides, the proposed FDDB OFDM radio receiver suffers from $\mathrm{RF}$ impairments, such as I/Q imbalance, which causes undesired mutual signal leakage significantly degrading the performance of reception. In order to implement a high performance FDDB OFDM radio transceiver, it is necessary to reduce the self-interference to a tolerable level and then estimate and compensate the I/Q imbalance.

\section{A) State-of-the-Art of Self-Interference Cancellation for Full-Duplex Wireless}

To handle the strong self-interference, [6-10] propose interference nulling from the perspective of antennas. In [11], a passive self-interference cancellation including directional isolation, absorptive shielding and cross polarization is proposed.

Furthermore, $[2,3,7,8,12]$ study the active self-interference cancellation (ASIC). In the following, these three approaches are presented in detail.

\section{1) Antenna Cancellation}

In [6], a three-antenna system using one receive antenna and two transmit antennas with $180^{\circ}$ phase shift is proposed. Specifically, two transmit antennas are placed symmetrically relative to the receive antenna. One of the two transmit signals is phase shifted internally by a fixed $\pi$ phase shifter. Then, the two received signals will add destructively at the receiver antenna. This original method is based on the symmetric placement of the transmit antennas relative to the receive antenna. A similar method is proposed by [9]. This antenna cancellation consists of three cancellation parts: (i) Transmit Antenna Cancellation - as implemented in [6]; (ii) Receive Antenna Cancellation - The two receive antennas are placed symmetrically relative to the transmit antenna. One of the two received signals is phase shifted internally by a fixed $\pi$ phase shifter. Then, the two received RF signals will add destructively at the RF adder. (iii) Antenna Cancellation which combines the transmit antenna cancellation and receive antenna cancellation to realize two stages of self-interference cancellation. In order to implement the antenna cancellation, the two receive antennas are placed symmetrically on the perpendicular bisector of the two transmit antennas. Antenna cancellation can achieve $45 \mathrm{~dB}$ self-interference cancellation, which has been experimentally demonstrated in [9], in wireless environment with few multiple paths. Another similar concept is proposed in [8], 
the full-duplex radio node employs three antennas, among which two antennas are used for transmission and the other one is used for reception. Besides, the radio node is equipped with two RF front-ends. One of the RF front-end is used for up-converting the baseband signal to RF signal for transmission. The other one is used for down-converting the received RF signal to baseband signal. In order to make the two RF signals from its own two transmit antennas be added destructively, the receive antenna is placed such that the distances from the two transmit antennas differ by half of the wavelength of the carrier frequency instead of using internal fixed $\pi$ phase shifter. The experimental results in [8] show that this antenna cancellation achieve $30 \mathrm{~dB}$ of self-interference cancellation. However, this scheme can only be used for narrowband signals because of the proportionality to the wavelength.

\section{2) Passive Self-Interference Suppression}

The basic principle of Passive Self-Interference Suppression (PSIS) is to use electromagnetical isolation techniques to reduce the power of the leaked signal from its own transmission to its own reception. The PSIS includes antenna separation, directional antenna, absorptive shielding and antenna polarization. Antenna separation is one of the simplest ways of PSIS, which could result in around $40 \mathrm{~dB}$ self-interference suppression when the antennas are separated by $6 \sim 8$ inches. Directional antenna could result in $36 \sim 38 \mathrm{~dB}$ self-interference suppression depending on the configuration [11]. Absorptive shielding can provide up to $25 \mathrm{~dB}$ absorption [11]. The antenna polarization in the near-field wireless communications could achieve $10 \mathrm{~dB}$ more self-interference isolation [9].

\section{3) Active Self-Interference Cancellation}

Active Self-Interference Cancellation (ASIC) is a method exploiting the knowledge of its own transmission and emulating the over-the-air self-interference channel to craft a cancellation signal and then subtract it in the receiver chain. Based on the SIC being implemented before or after the Analog-to-Digital Converter (ADC), the ASIC is classified as Active Analog Self-Interference Cancellation (AASIC) and Active Digital Self-Interference Cancellation (ADSIC).

(I) Active Analog Self-Interference Cancellation: As the name suggests, the AASIC is the active cancellation performed in analog domain before the received signal passes through the ADC. Based on the SIC being carried out in the RF domain or Base Band (BB) domain, the AASIC is divided into Active Analog RF Self-interference Cancellation (AARFSIC) $[2,3,7]$ and Active Analog BB Self-Interference Cancellation (AABBSIC) [13]. In [3], a Balun is used to obtain the inverse version of the transmitted signal which can be used as the reference signal to cancel the self-interference. The Received Signal Strength Indicator (RSSI) is used to adjust the attenuation and delay which emulates the over-the-air self-interference channel. In fact, the full-duplex radio node knows what it transmits and therefore it is easy for a it to obtain the estimated coefficients of the wireless self-interference channel. Besides, OFDM converts a frequency selective fading channel into a large number of parallel frequency flat fading sub-channels which could help implement the self-interference cancellation in each sub-carrier channel. Therefore, [2] utilizes another transmitter chain to craft the RF cancellation signal and then implements the self-interference cancellation at the RF stage. 
(II) Active Digital Self-Interference Cancellation: The ADSIC aims at cleaning out the cancelable residual self-interference in the digital domain after the PSIS and AASIC.

\section{B) Related Works on the I/Q Imbalance Estimation and Compensation}

There are mainly two different RF architectures to convert the RF signals to baseband signals, i.e. through intermediate frequency (IF) or via direct-conversion (zero-IF). Based on this, radio receivers are classified into zero-IF receivers and superheterodyne (with IF) receivers. With respect to different kinds of radio receivers, there are different approaches for addressing the I/Q imbalance estimation and compensation. Due to the lot of approaches on that topic, we will just point out some significant examples.

- For Zero-IF Receivers

- Based on Training Symbols

The authors in [14] proposed an I/Q imbalance estimation technique based on the design of special pilot tones and a least mean squares (LMS) algorithm. This technique requires the modification of the training sequences and suffers from a huge number of OFDM training symbols and a low convergence rate. [15] proposed a special training sequence design for the I/Q imbalance parameter estimation. Another I/Q imbalance estimation technique based on the redesign of the preamble proposed by [16] requires the modification of the preamble of IEEE 802.11a. [17] and [18] proposed an I/Q imbalance estimation and compensation based on the assumption that the adjacent subcarriers are highly correlated.

- Based on Data Symbols

Windisch et al. [19] proposed the I/Q imbalance estimation based on the statistical analysis of data symbols, i.e. blind estimation. This method requires a large number of data symbols to obtain a good I/Q imbalance estimation.

- For Superheterodyne Receiver

- Based on Training Symbols

[20] proposed to employ two different long training symbols instead of two identical long training symbols to carry out the channel and I/Q imbalance estimation.

However, the two long training symbols are identical in the practical implementation of IEEE $802.11 \mathrm{~g}$. The authors further proposed an iterative algorithm by both using the training and data OFDM symbols.

- Based on Data Symbols

The blind I/Q imbalance estimation based on the two received baseband signals (uncompensated desired and image signal) were presented in [21] and [22]. This approach relies on the assumption that the desired and image signals are zero-mean wide-sense stationary random processes and mutually uncorrelated, which puts high requirements on the design of the signals. 


\section{C) Contribution}

In this paper, a FDDB superheterodyne receiver based on the RF front-end [1] is proposed and studied. The strong self-interference encountered in the FDDB OFDM radio is successfully managed to the noise level by using a simple Band Pass Filter (BPF) and the available AASIC method when the self-interference channel is assumed to be one-path, one-delay channel. However, the I/Q imbalance in the FDDB RF front-end significantly degrades the reception performance of the proposed radio system. The available I/Q imbalance estimation and compensation methods are based on the signal processing of one signal with one carrier frequency, which are not suitable to address the $\mathrm{I} / \mathrm{Q}$ imbalance faced in this flexible radio receiver. This is because each receiver chain will receive two signals even if they share a common center frequency. Hence, the I/Q imbalance parameters should be obtained by cooperatively processing two received signals. Therefore, we developed a suitable and advanced I/Q imbalance estimation and compensation method for mitigating the I/Q imbalance existing in the FDDB RF front-end. This method is based on a simple digital signal processing of the two estimated self-interference channel coefficients, which renders it a practical I/Q imbalance estimation and compensation method.

The rest of the paper is organized as follows: Section II presents the proposed radio transceiver architecture including the general system model, self-interference cancellation model in full-duplex radio and the architecture of dual-band RF front-end. The I/Q imbalance estimation and compensation are given in Section III. Simulation results are presented in Section IV. Finally, the conclusion and the perspectives of this work are given in Section $\mathrm{V}$.

\section{Wideband Full-Duplex Dual-Band OFDM Radio Transceiver}

\section{A) System Model}

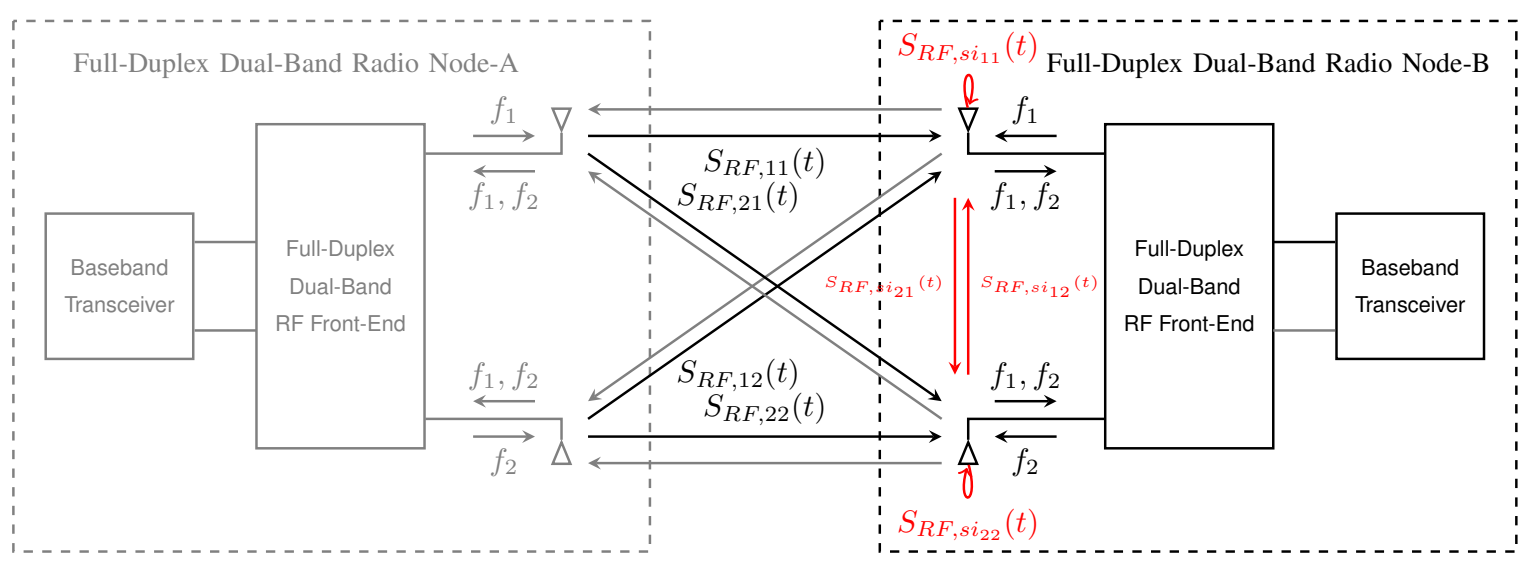

Figure 1: Block diagram of the full-duplex dual-band wireless radios.

This section presents the general system model of the proposed flexible radio system as shown in Fig. 1 and the details of the FDDB OFDM radio node as shown in Fig. 2. Each of the proposed radio nodes employs two antennas, which are compatible with future 


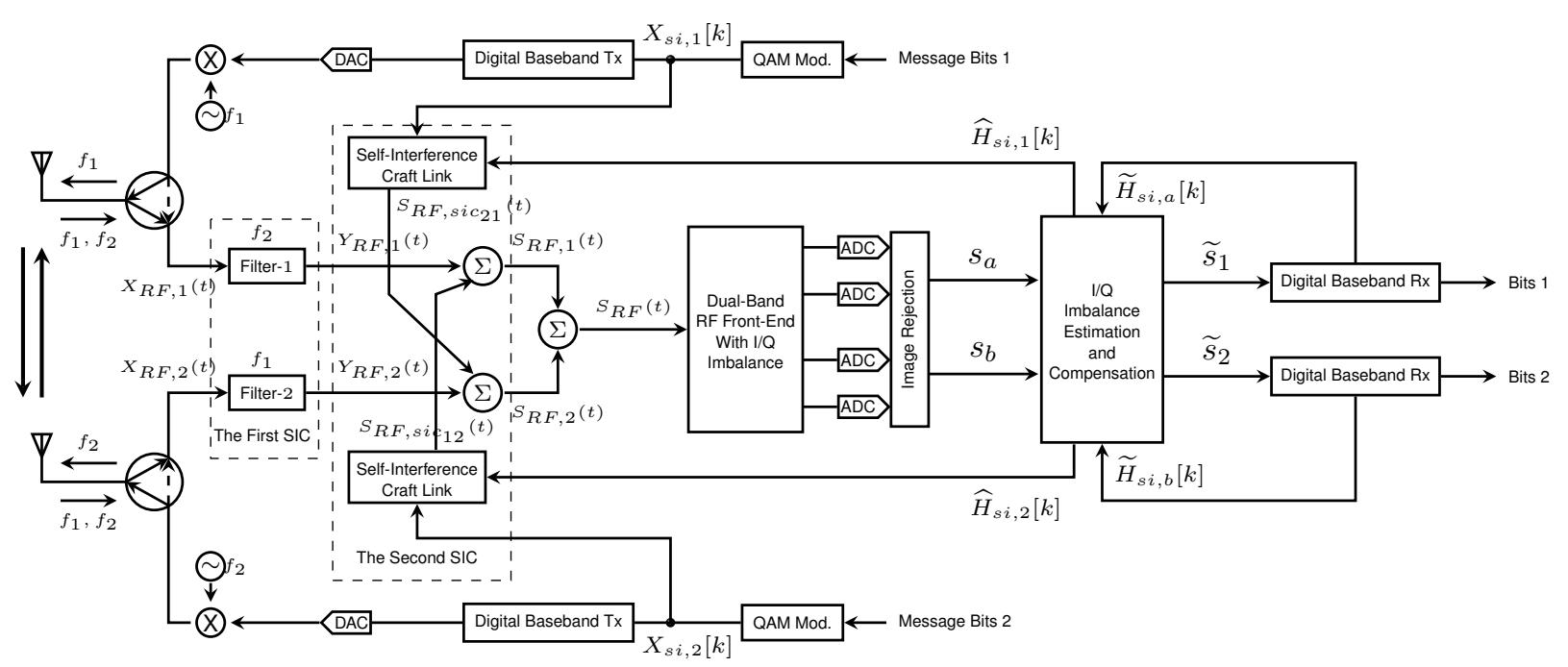

Figure 2: Full-duplex dual-band OFDM radio transceiver.

multi-antenna systems. Each antenna is used for simultaneous transmission and reception via a circulator $[23,24]$ separating the input and output signals. As this problem is symmetric, the radio node-B as shown in Fig. 1 is only studied. At the FDDB radio node- $\mathrm{B}$, the first antenna transmits the RF signal with carrier frequency $f_{1}$ and receives mixed $\mathrm{RF}$ signals including the $\mathrm{RF}$ signals from the distant radio node (FDDB radio node-A) with carrier frequency $f_{1}$ and $f_{2}$ and the RF signals from the local radio node (radio node-B) with carrier frequency $f_{2}$. Therefore, the signals received by the $i^{t h}$ antenna of the local radio node plus the self-interference signal leaked from the circulator can be expressed as:

$$
X_{R F, i}(t)=S_{R F, i j}(t)+S_{R F, i i}(t)+S_{R F, s i_{i j}}(t)+a S_{R F, s i_{i i}}(t), \forall\{i, j\} \in A
$$

where $S_{R F, i j}(t)$ and $S_{R F, i i}(t)$ are the RF signals emitted from the $j^{\text {th }}$ antenna and the $i^{\text {th }}$ antenna of the distant radio node respectively and received by the $i^{\text {th }}$ antenna of the local radio node; $S_{R F, s i_{i j}}$ denotes the internal $R F$ self-interference signal transmitted from the $j^{\text {th }}$ antenna and received by the $i^{\text {th }}$ antenna of the local radio node; $S_{R F, s i_{i i}}$ is the inner RF self-interference signal leaked from its own transmission, $a$ being the inner-SI factor including multi-path reflection factor and inner leakage factor of the circulator and $A \triangleq\{i \in[1,2], j \in[1,2], i+j=3\}$. Due to the short distance between the antennas of the same radio node, the internal self-interference $S_{R F, s i_{i j}}$ is much stronger than the signals from the distant radio node, e.g. $90 \sim 110 \mathrm{~dB}$ higher $[23,25]$. With respect to the inner self-interference, it is even stronger than the internal self-interference which relies on the fact that the circulator has only $15 \mathrm{~dB}$ of isolation [23]. Therefore, the reception of the desired signal from the distant radio node is challenged by these two strong self-interference signals.

After each circulator, two self-interference cancellation stages are implemented to eliminate the inner self-interference signal leaked from its own transmission and reduce the internal self-interference signal from the other antenna of the local radio node. 


\section{B) Self-Interference Cancellation}

If no measures are taken, the strong self-interference brought by the FDDB radio could saturate the receiver chain due to the fact that the ADC has a limited dynamic range. Therefore, it is necessary to suppress or reduce these self-interference signals to a tolerable level in order to obtain the expected system performance. In order to combat the inner and internal self-interference, two different self-interference cancellation methods are implemented.

\section{1) The First Self-Interference Cancellation}

The first self-interference cancellation is carried out by using BPF- $i$ (Band Pass Filter, perfect BPFs are assumed) with center frequency $f_{j}$ to filter out the inner RF self-interference signal $a S_{R F, s i_{i i}}$ induced by near-field reflection and the imperfect circulator isolation, as well as the RF signal $S_{R F, i i}(t)$ with carrier frequency $f_{i}$ from the $i^{\text {th }}$ antenna of the distant radio node. Then, the RF signals with carrier frequency $f_{j}$ including the signal of interest $S_{R F, i j}(t)$ and the over-the-air self-interference signal $S_{R F, s i_{i j}}(t)$ can be represented by

$$
Y_{R F, i}(t)=S_{R F, i j}(t)+S_{R F, s i_{i j}}(t), \forall\{i, j\} \in A
$$

\section{2) The Second Self-Interference Cancellation}

In order to cancel the internal self-interference signal $S_{R F, s i_{i j}}(t)$ expressed in $(2),[3,26]$ proposed active self-interference cancellation and [11] studied the passive self-interference suppression. In this paper, we craft the RF cancellation signal as presented in [27] and carry out the cancellation at the RF stage as shown in Fig. 3.

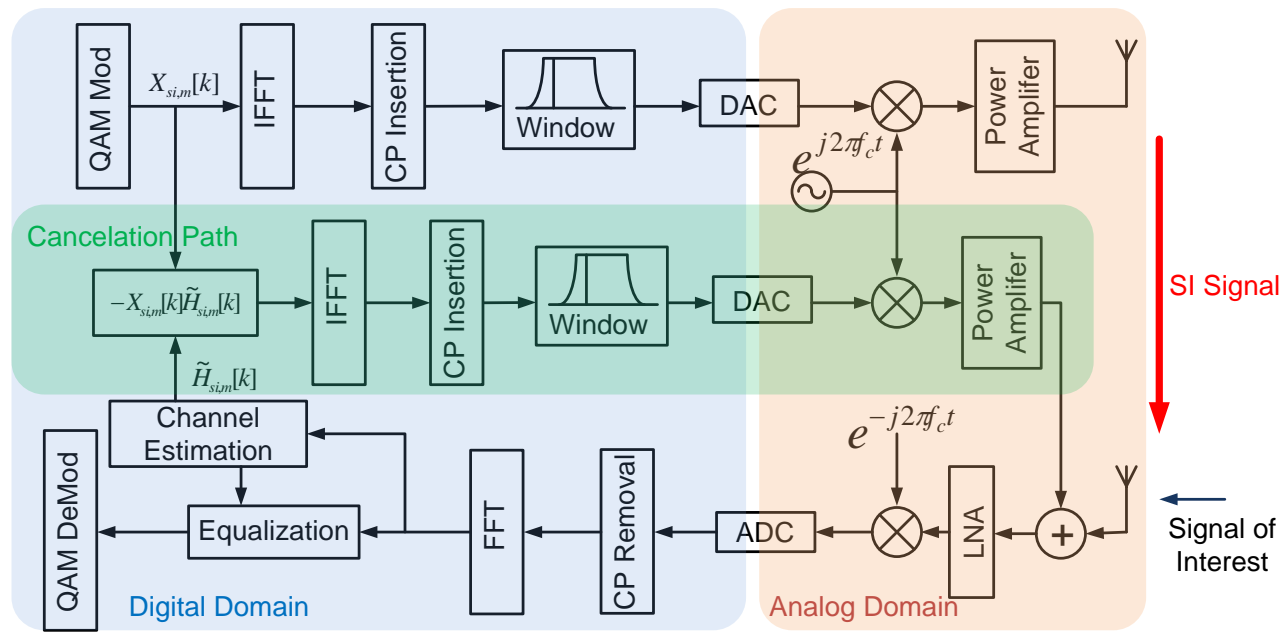

Figure 3: Architecture of self-interference cancellation for in-band two-antenna Full-Duplex wireless.

The system studied here is an OFDM based IEEE 802.11g WLAN with $N=64$ subcarriers $\left(N_{n z}=52\right.$ non-zeros subcarriers). In each frame of IEEE $802.11 \mathrm{~g}$, the preamble consists of 10 identical short OFDM symbols (each of length 16) and two 
identical long training symbols (each of length 64). Each long training signal is generated via IFFT of 52 known BPSK symbols $T_{k}, k \in\left[1, N_{n z}\right]$ and 12 zeros.

Perfect frequency and timing synchronization are assumed in this paper. Therefore, at the receiver side, after the down-conversion of the RF signal received and removal of the cyclic prefix, the baseband frequency domain signal can be obtained by the FFT of the time domain baseband signal. The $m^{\text {th }}$ demodulated OFDM symbol can be expressed as

$$
\begin{aligned}
R_{i, m}[k]= & \sqrt{P_{j}} \sqrt{L_{j}} \cdot X_{j, m}[k] H_{i j}[k]+\sqrt{P_{s i, j}} \sqrt{L_{s i, j}} \cdot X_{s i, j, m}[k] H_{s i, i j}[k]+Z_{m}[k], \\
& 1 \leq k \leq N_{n z}, \forall\{i, j\} \in A
\end{aligned}
$$

where $P_{j}$ and $P_{s i, j}$ represent the transmit power of the signal of interest and self-interference signal respectively, $L_{j}$ and $L_{s i, j}$ are the propagation loss of the intended radio link and self-interference radio link, $X_{j, m}[k]$ and $X_{s i, j, m}[k]$ denote the transmit symbol carried by the $k^{t h}$ subcarrier from the distant radio node and local radio node, $H_{i j}[k]$ and $H_{s i, i j}[k]$ represent the transfer function of the $k^{t h}$ subcarrier channel of the distant radio link and self-interference radio link. $Z_{m}[k]$ corresponds to the frequency domain expression of the thermal noise and follows the normal distribution $\mathcal{N}\left(0, \delta_{n}^{2}\right), N_{n z}$ denotes the number of nonzero subcarriers. $X_{j, m}[k]$ can be treated as mutually independent random variables independent of $H_{i j}[k] . X_{s i, j, m}[k]$ has the same characteristics as $X_{j, m}[k]$. Without loss of generality, the transfer function $H_{i j}[k]$ and $H_{s i, i j}[k]$ are normalized and with average channel gain $E\left\{\left|H_{i j}[k]\right|^{2}\right\}=E\left\{\left|H_{s i, i j}[k]\right|^{2}\right\}=1$. From (3), the self-interference signal can be canceled if the Channel State Information (CSI) of the self-interference channel is available because the transmit symbols $X_{s i, j, m}[k]$ are known. Therefore, the self-interference channel estimation is carried out in the following subsection before implementing the second self-interference cancellation.

Self-Interference Channel Estimation: In IEEE 802.11g, the channel estimation is

Radio Node-A

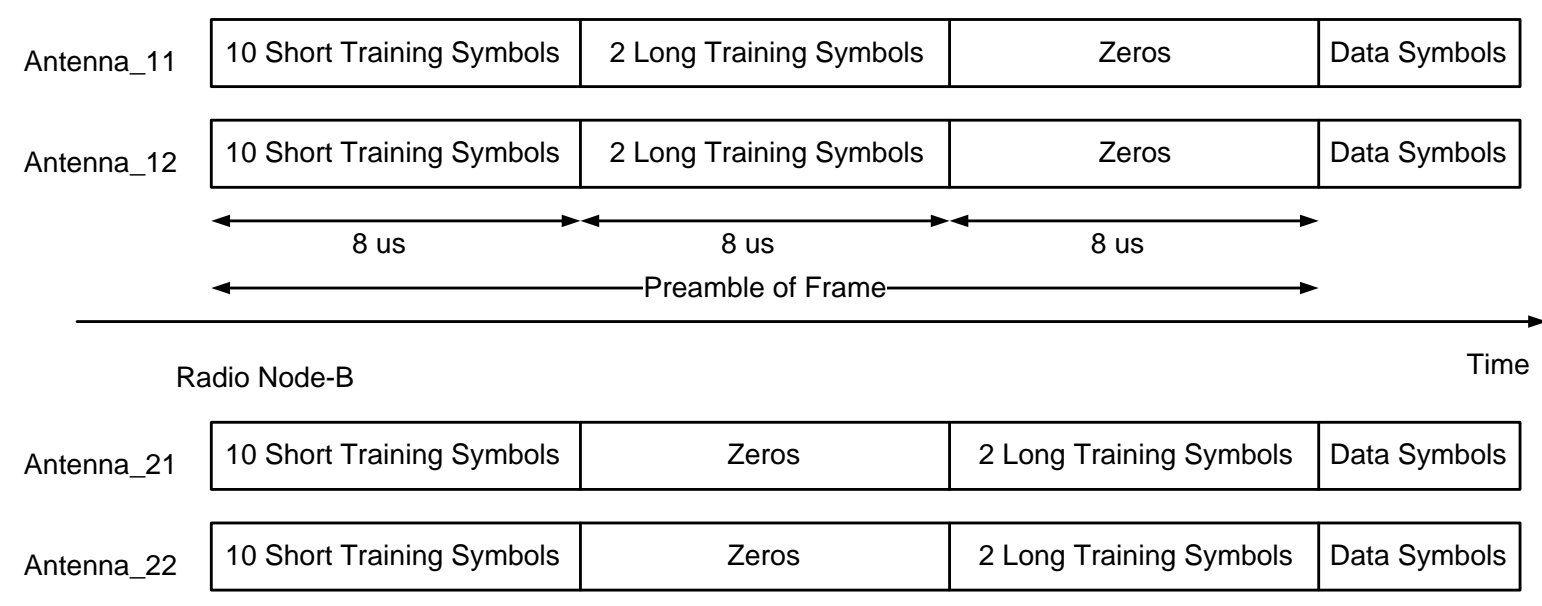

Figure 4: Frame structure for the Full-Duplex Dual-Band IEEE 802.11g.

carried out by dividing the received demodulated long training symbols by the pilot pattern with the known BPSK symbol sequences $T[k], k \in\left[1, N_{n z}\right]$ with $E\left\{|T[k]|^{2}\right\}=1$. In order to avoid the self-interference channel estimation error caused by the signal of interest, the long training signals from different radio nodes are appointed to be 
transmitted at different time slots. Namely, the frame structure is modified as shown in Fig. 4. Therefore, the estimated channel transfer function after normalization is:

$$
\widetilde{H}_{s i, i j}[k]=H_{s i, i j}[k]+\underbrace{Z[k] /\left(\sqrt{P_{s i, j}} \sqrt{L_{s i, j}} T[k]\right)}_{\text {channel estimation error due to thermal noise }}, \forall\{i, j\} \in A
$$

The second term of the right side of the equation (4) is denoted as the channel estimation error of the $k^{t h}$ subcarrier channel due to the thermal noise.

Self-Interference Cancellation: With the estimated coefficients of the self-interference channel available, the crafted RF cancellation signal as presented in [27] is represented by

$$
S_{R F, s i c_{i j}}(t)=\Re\left(\sum_{k=-\frac{N}{2}}^{\frac{N}{2}-1} X_{s i, j}[k] \widetilde{H}_{s i, i j}[k] e^{j 2 \pi \Delta f k t} e^{j 2 \pi f_{j} t}\right), \forall\{i, j\} \in A
$$

where $\Re(\cdot)$ denotes the real part of $(\cdot), X_{s i, j}[k]$ is the complex symbol carried by the $k^{t h}$ subcarrier and to be emitted from the $j^{\text {th }}$ antenna of the local radio node, $\widetilde{H}_{s i, i j}[k]$ represents the estimated coefficient of $k^{\text {th }}$ subcarrier channel of the over-the-air self-interference channel from the $j^{\text {th }}$ antenna to the $i^{t h}$ antenna of the local radio node, and $\Delta f$ denotes the subcarrier space.

With the RF cancellation signal available, the second self-interference cancellation is carried out via subtracting the RF signal $Y_{R F, i}(t)$ by the crafted RF cancellation signal $S_{R F, s i c_{i j}}(t)$ at the RF adder stage. After that, the remaining signals to be input to the dual-band RF front-end is expressed as

$$
\begin{aligned}
S_{R F, i}(t) & =Y_{R F, i}(t)-S_{R F, s i c_{i j}}(t),\{i, j\} \in A \\
& =S_{R F, i j}(t)+\underbrace{S_{R F, s i_{i j}}(t)-S_{R F, s i c_{i j}}(t)}_{\text {residual self-interference }}
\end{aligned}
$$

Therefore, the RF signal $S_{R F, i}(t)$ to be input into the dual-band RF front-end comprises the desired signal $S_{R F, i j}(t)$ from the distant radio node and the residual self-interference. In order to quantify the impact of the residual self-interference on the proposed radio transceiver, the power of the residual self-interference will be calculated in the following subsection.

Residual Self-Interference: Although the self-interference cancellation is implemented at the RF component, the residual self-interference can be calculated and analyzed in the frequency domain. Therefore, we can equivalently understand that the self-interference cancellation is done by subtracting the cancellation signal $\sqrt{P_{s i, j}} \sqrt{\widetilde{L}_{s i, j}} X_{s i, j, m}[k] \widetilde{H}_{s i, i j, m}[k]$ from the self-interference signal received in the frequency domain. After the interference cancellation, the residual self-interference can be represented by

$$
\begin{aligned}
R_{i, m, r e s_{s i}}[k]= & \sqrt{P_{s i, j}} \sqrt{L_{s i, j}} X_{s i, j, m}[k] H_{s i, i j}[k] \\
& -\sqrt{P_{s i, j}} \sqrt{\widetilde{L}_{s i, j}} X_{s i, j, m}[k] \widetilde{H}_{s i, i j}[k], \forall\{i, j\} \in A
\end{aligned}
$$


$L_{s i, j}$, denoting the large-scale fading of the self-interference link, can be obtained or calculated by previous measurements. Therefore, $\widetilde{L}_{s i, j}=L_{s i, j}$ can be guaranteed. Then, the residual self-interference can be simplified as

$$
R_{i, m, r e s_{s i}}[k]=-\frac{X_{s i, j, m}[k]}{T[k]} Z_{m}^{\prime}[k]
$$

Because of the independence between $X_{s i, j, m}[k]$ and $T[k]$ in (8), the power of the residual self-interference is

$$
P_{i, s i, r e s i d u a l}=E\left[\left|R_{i, m, r e s_{s i}}[k]\right|^{2}\right]=\delta_{n}^{2}
$$

From (9), the power of residual self-interference after self-interference cancellation is equal to that of the receiver thermal noise [28]. Therefore, the residual self-interference can be regarded as another additive thermal noise superimposed on the desired signal.

\section{C) Dual-Band RF Front-End}

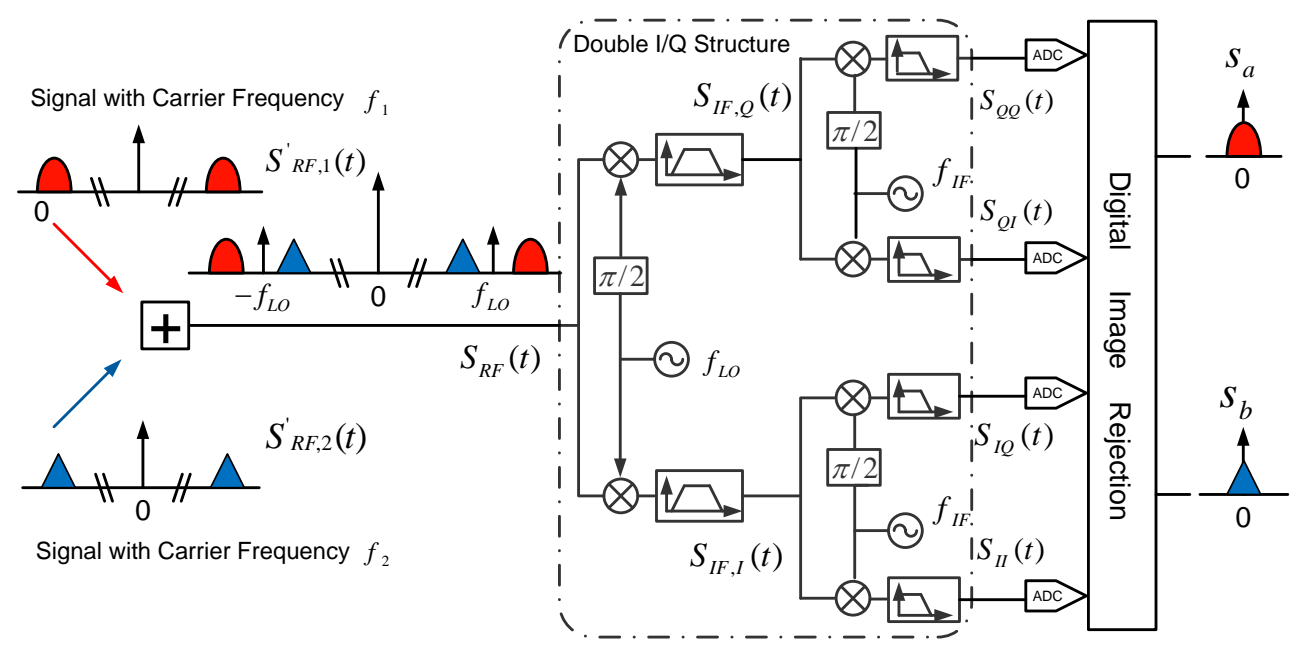

Figure 5: Architecture of Dual-Band RF front-end.

The two received RF signals $S_{R F, 1}^{\prime}(t) \triangleq S_{R F, 2}(t)$ with carrier frequency $f_{1}$ and $S_{R F, 2}^{\prime}(t) \triangleq S_{R F, 1}(t)$ with carrier frequency $f_{2}$, after self-interference cancellation, will be down-converted to baseband signal by utilizing the double I/Q structure as shown in Fig. 5 .

\section{1) Dual-Band RF Front-End without I/Q Imbalance}

In order to facilitate the theoretical study of the dual-band RF front-end, $s_{1}=I_{1}+\jmath Q_{1}$ and $s_{2}=I_{2}+\jmath Q_{2}\left(\jmath^{2}=-1\right)$ are used to denote the received equivalent baseband complex symbols carried by the carrier frequencies $f_{1}$ and $f_{2}$. Then, the RF signals $S_{R F, 1}^{\prime}(t)$ and $S_{R F, 2}^{\prime}(t)$ at the input of the double I/Q structure are:

$$
\begin{aligned}
& S_{R F, 1}^{\prime}(t)=I_{1}(t) \cos \left(2 \pi f_{1} t\right)-Q_{1}(t) \sin \left(2 \pi f_{1} t\right) \\
& S_{R F, 2}^{\prime}(t)=I_{2}(t) \cos \left(2 \pi f_{2} t\right)-Q_{2}(t) \sin \left(2 \pi f_{2} t\right)
\end{aligned}
$$


Note that the signal model in (10) and (11) is reasonably valid even for an interference and noisy environment because $I_{i}(t)$ can represent the sum of the real part of the received symbol, including the desired data and the residual self-interference data symbol, and noise, $Q_{i}(t)$ can represent the sum of the image part of the received symbol including desired data and residual self-interference data symbol and the receiver thermal noise. As shown in Fig. 5, after the mixed signal $S_{R F}(t)=S_{R F, 1}^{\prime}(t)+S_{R F, 2}^{\prime}(t)$ going through the first frequency translation stage with carrier frequency $f_{L O}=\left(f_{1}+f_{2}\right) / 2$, the band pass signal is filtered out. Then, the I/Q branch of intermediate frequency (IF) signal can be obtained as

$$
\begin{aligned}
& S_{I F, I}(t)=\frac{I_{1}(t)+I_{2}(t)}{2} \cos \left(2 \pi f_{I F} t\right)+\frac{-Q_{1}(t)+Q_{2}(t)}{2} \sin \left(2 \pi f_{I F} t\right) \\
& S_{I F, Q}(t)=\frac{I_{1}(t)-I_{2}(t)}{2} \sin \left(2 \pi f_{I F} t\right)+\frac{Q_{1}(t)+Q_{2}(t)}{2} \cos \left(2 \pi f_{I F} t\right)
\end{aligned}
$$

where $f_{I F}=\left(f_{1}-f_{2}\right) / 2=f_{1}-f_{L O}=f_{L O}-f_{2}$.

The baseband signals $s_{I I}(t), s_{I Q}(t), s_{Q I}(t)$ and $s_{Q Q}(t)$ obtained by down-converting the IF signals $S_{I F, I}(t)$ and $S_{I F, Q}(t)$ with carrier frequency $f_{I F}$ can be expressed as

$$
\begin{aligned}
s_{I I}(t) & =\frac{I_{1}(t)+I_{2}(t)}{4} \\
s_{I Q}(t) & =\frac{Q_{1}(t)-Q_{2}(t)}{4} \\
s_{Q I}(t) & =\frac{Q_{1}(t)+Q_{2}(t)}{4} \\
s_{Q Q}(t) & =\frac{-I_{1}(t)+I_{2}(t)}{4}
\end{aligned}
$$

After the analog-to-digital converter (ADC), $s_{I I}=\frac{I_{1}+I_{2}}{4}, s_{I Q}=\frac{Q_{1}-Q_{2}}{4}$, $s_{Q I}=\frac{Q_{1}+Q_{2}}{4}, s_{Q Q}=\frac{-I_{1}+I_{2}}{4}$ can be obtained. Then, using digital-image-rejection, the received baseband complex symbols $s_{a}$ and $s_{b}$ corresponding to symbols $s_{1}$ and $s_{2}$ are given by

$$
\begin{aligned}
s_{a} & =s_{I I}-s_{Q Q}+\jmath\left(s_{I Q}+s_{Q I}\right) \\
& =\frac{I_{1}+\jmath Q_{1}}{2} \\
& =\frac{s_{1}}{2} \\
s_{b} & =s_{I I}+s_{Q Q}+\jmath\left(-s_{I Q}+s_{Q I}\right) \\
& =\frac{I_{2}+\jmath Q_{2}}{2} \\
& =\frac{s_{2}}{2}
\end{aligned}
$$

From (18) and (19), the two different kinds of signals (even with different physical layer technologies) are perfectly separated by using double I/Q structure. That means that the dual-band radio receiver can simultaneously process two different kind of signals with different carrier frequencies by using a relative simple RF front-end compared to 
conventional multiband radios. However, this noticeable flexibility can be obtained only when the RF front-end is theoretically ideal. In the practical implementation of wireless system, any part of the I/Q imbalance of the RF front-end may contribute to degrade the radio receiver performance and this is no exception for the FDDB OFDM radio. In the following subsection 2), the I/Q imbalance model and its impact on the FDDB OFDM radio receiver will be studied.

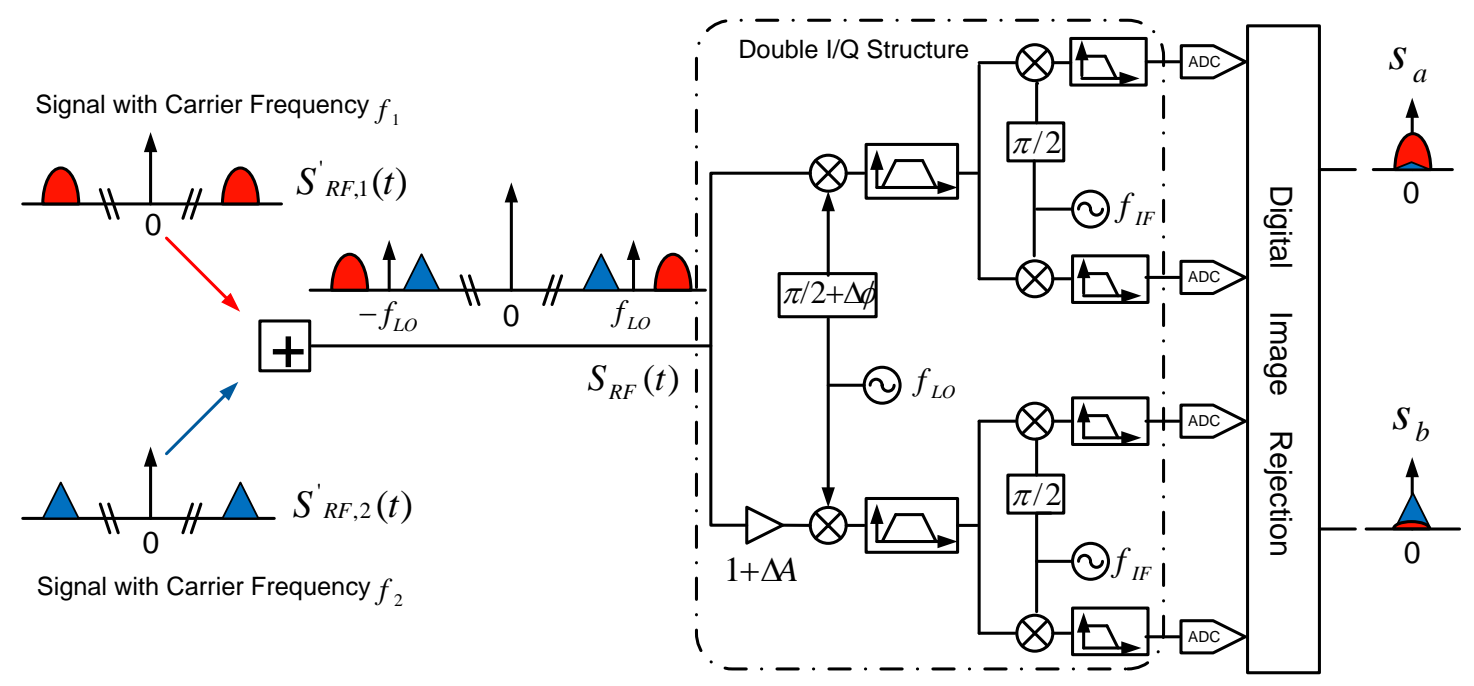

Figure 6: Dual-Band RF front-end with I/Q imbalance

\section{2) Dual-Band RF Front-End with I/Q Imbalance}

The purpose of a radio front-end is to down-convert the RF signal to baseband analog signal. However, all of the analog components (such as mixers, filters and ADC) at the radio front-end lead to deviations from the desired $90^{\circ}$ phase shift and the desired equal gain in the I and $\mathrm{Q}$ branches as shown in Fig. 6. Here, $\Delta A$ and $\Delta \phi$ are used to denote the amplitude imbalance and the phase imbalance respectively. ${ }^{1}$ In dual-band radio receivers, this I/Q imbalance causes undesirable mutual signal leakage. After double I/Q frequency translation with I/Q imbalance, the two baseband analog signals can be expressed as [4]

$$
\begin{aligned}
& s_{a}=\alpha s_{1}+\beta s_{2}^{*} \\
& s_{b}=\alpha s_{2}+\beta s_{1}^{*}
\end{aligned}
$$

where $s_{a}$ and $s_{b}$ are the two output signals from the double I/Q structure with I/Q imbalance, $(\cdot)^{*}$ denotes complex conjugation, and the I/Q imbalance coefficients $\alpha$ and $\beta$ [4] are represented by

$$
\begin{aligned}
& \alpha=\frac{1+(1+\Delta A) e^{-\jmath \Delta \phi}}{2} \\
& \beta=\frac{1-(1+\Delta A) e^{+\jmath \Delta \phi}}{2}
\end{aligned}
$$

\footnotetext{
${ }^{1}$ Here, the I/Q imbalance is just modeled in the first IQ demodulator due to fact that I/Q imbalance in the second IQ demodulator has negligible effect compared to that of the first one [4].
} 
According to the definition (22) (23), we get

$$
\alpha+\beta^{*}=\alpha^{*}+\beta=1
$$

Especially, with an ideal I/Q, i.e. $\Delta A=0$ and $\Delta \phi=0$, therefore $\alpha=1$ and $\beta=0$, which means the received two different carrier frequency signals are separated completely by using double I/Q structure just as the study case in subsection 1).

From the equations (20) and (21), the I/Q imbalance makes the image of the other signal superimpose on the desired signal. This mutual leakage of signals will be regarded as interference to degrade the performance of the radio reception. However, if the I/Q imbalance parameters $\left(\alpha\right.$ and $\beta$ ) are known, $s_{1}$ and $s_{2}$ can be calculated as

$$
\begin{aligned}
& s_{1}=\frac{\alpha^{*} s_{a}-\beta s_{b}^{*}}{|\alpha|^{2}-|\beta|^{2}} \\
& s_{2}=\frac{\alpha^{*} s_{b}-\beta s_{a}^{*}}{|\alpha|^{2}-|\beta|^{2}}
\end{aligned}
$$

That means, even with I/Q imbalance, the two received signals can be separated completely just like the RF front-end without I/Q imbalance does if the values of $\alpha$ and $\beta$ are known. Unfortunately, the exact values of $\alpha$ and $\beta$ are difficult to obtain. However, $\alpha$ and $\beta$ are quite stable due to the reason that the values of $\Delta A$ and $\Delta \phi$ are dependent on the performance of analog devices in the RF front-end. Therefore, the values can be estimated by using a training sequence.

In order to mitigate the I/Q imbalance existing in the FDDB RF front-end, the algorithm of the I/Q imbalance estimation and compensation will be presented in section III.

\section{D) Complexity Comparison}

The proposed FDDB OFDM radio is based on a cooperative combination of full-duplex OFDM radio and dual-band RF front-end. Compared to the conventional half-duplex radio system, the full-duplex radio needs an auxiliary radio link to craft an RF cancellation signal, which requires some additional hardware components [27]. Dual-band $\mathrm{RF}$ front-end could significantly reduce the system complexity and power consumption compared to the current multimode radio system [4]. Therefore, the flexible FDDB OFDM radio could realize a multimode radio with less complexity compared to the conventional multimode radio, but only at a cost of additional hardware components.

\section{I/Q Imbalance Estimation and Compensation}

\section{A) I/Q Imbalance Estimation}

In our design, the $\mathrm{I} / \mathrm{Q}$ imbalance estimation is carried out during the self-interference channel estimation. Therefore, the received symbols $s_{1}$ and $s_{2}$ in (20) and (21) just include the long training symbols from its own transmitter and the thermal noise during the I/Q imbalance estimation because different radio nodes take different time slots for transmitting the long training signals. Therefore, these two equations can be rewritten 
during the time of self-interference channel estimation as

$$
\begin{aligned}
& s_{l t s, a}=\alpha s_{l t s_{s i}, 12}+\beta s_{l t s_{s i}, 21}^{*} \\
& s_{l t s, b}=\alpha s_{l t s_{s i}, 21}+\beta s_{l t s_{s i}, 12}^{*}
\end{aligned}
$$

where $s_{l t s_{s i}, 12}$ is the long training symbols transmitted from the $2^{\text {nd }}$ antenna and received by the $1^{s t}$ antenna of the local radio node, and $s_{l t s_{s i}, 21}$ is the long training symbols from the $1^{\text {st }}$ antenna and received by the $2^{\text {nd }}$ antenna of the local radio node.

According to (27) and (28), and using the least squares (LS) channel estimation algorithm in the frequency domain, the estimated coefficients of the self-interference channel-1 $H_{s i, a}$ and channel-2 $H_{s i, b}$ can be expressed as

$$
\begin{aligned}
& H_{s i, a}[k]=\alpha \widetilde{H}_{s i, 12}[k]+\beta T^{\prime}[k] \cdot \widetilde{H}_{s i, 21}^{*}\left[N_{n z}-k+1\right] \\
& H_{s i, b}[k]=\alpha \widetilde{H}_{s i, 21}[k]+\beta T^{\prime}[k] \cdot \widetilde{H}_{s i, 12}^{*}\left[N_{n z}-k+1\right]
\end{aligned}
$$

where $\widetilde{H}_{s i, 12}[k]$ denotes the coefficient of the $k^{t h}$ subcarrier channel of the self-interference channel from the $2^{\text {nd }}$ antenna to the $1^{\text {st }}$ antenna of the local radio node; $\widetilde{H}_{s i, 21}[k]$ denotes the coefficient of the $k^{t h}$ subcarrier channel of the self-interference channel from the $1^{\text {st }}$ antenna to the $2^{n d}$ antenna of the local radio node. $\widetilde{H}_{s i, 21}\left[N_{n z}-k+1\right]$ and $\widetilde{H}_{s i, 12}\left[N_{n z}-k+1\right]$ are the mirror images of the channel $\widetilde{H}_{s i, 21}[k]$ and $\widetilde{H}_{s i, 12}[k]$, $T^{\prime}[k] \triangleq T[k] T\left[N_{n z}-k+1\right]$ and "." denotes component-wise vector multiplication. (29) and (30) show that each channel estimation consists of two components: one is the self-interference channel which is scaled by a complex factor $\alpha \approx 1$ and the other one is the mirror image of the other self-interference channel scaled by factor $\beta T^{\prime}[k] \approx 0$. As a consequence, the I/Q imbalance in the double frequency structure makes the mirror image of the self-interference channel superimpose on the desired self-interference channel. This channel estimation error induced by I/Q imbalance will affect the equalization at the receiver.

In the FDDB wireless, the distance between the antennas of the same radio node is very short, so the power of the line-of-sight path dominates the self-interference channel. As a consequence, the self-interference channel is close to a frequency flat fading channel [11]. From (29) and (30), if $\alpha$ and $\beta$ are known exactly, the self-interference channel $\widetilde{H}_{s i, 12}[k]$ and $\widetilde{H}_{s i, 21}[k]$ can be obtained as

$$
\begin{aligned}
& \widetilde{H}_{s i, 12}[k]=\frac{\alpha^{*} H_{s i, a}[k]-\beta T^{\prime}[k] \cdot H_{s i, b}^{*}\left[N_{n z}-k+1\right]}{|\alpha|^{2}-|\beta|^{2}} \\
& \widetilde{H}_{s i, 21}[k]=\frac{\alpha^{*} H_{s i, b}[k]-\beta T^{\prime}[k] \cdot H_{s i, a}^{*}\left[N_{n z}-k+1\right]}{|\alpha|^{2}-|\beta|^{2}}
\end{aligned}
$$

The self-interference channel $\widetilde{H}_{s i, 12}[k]$ and $\widetilde{H}_{s i, 21}[k]$ are frequency flat fading which can be shown as in Fig. 7, while the estimated self-interference channel $H_{s i, a}[k]$ and $H_{s i, b}[k]$ with I/Q imbalance are more likely a frequency selective fading channel. With the estimated $\widetilde{\alpha}$ and $\widetilde{\beta}$ under the I/Q imbalance condition, the virtual self-interference channel $\widehat{H}_{s i, 12}[k]$ 


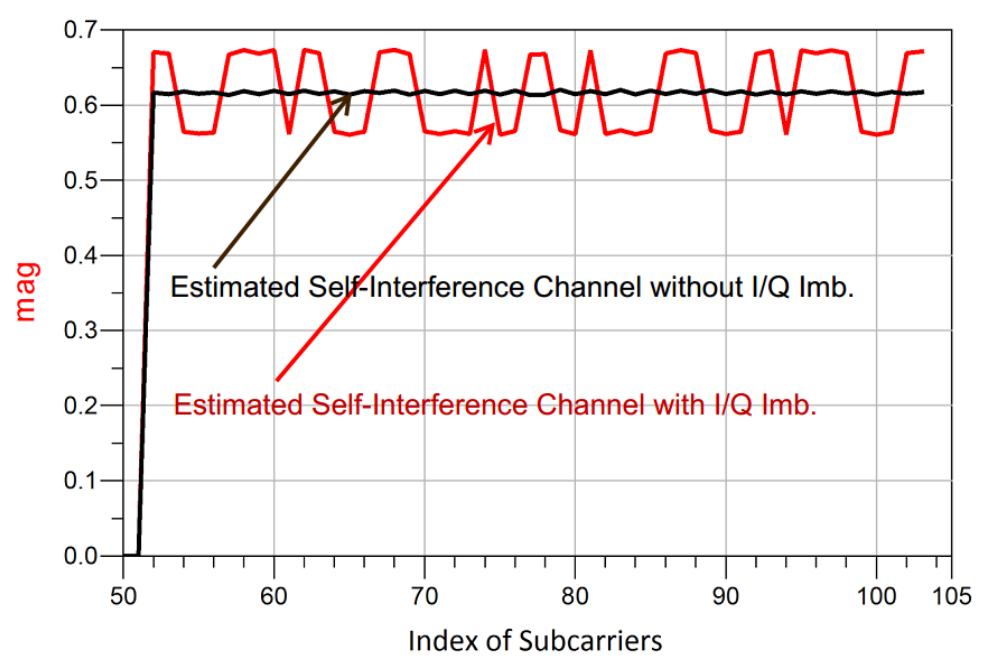

Figure 7: The estimated magnitude of each subcarrier channel of the over-the-air self-interference channel with or without I/Q Imbalance. Without I/Q imbalance, the estimated over-the-air selfinterference channel is almost a frequency flat fading channel, while this self-interference channel will be a frequency selective fading channel when I/Q imbalance $\left(\Delta A=0.2, \Delta \phi=10^{\circ}\right)$ exists in the full-duplex dual-band radio front-end.

and $\widehat{H}_{s i, 21}[k]$ can be obtained as

$$
\begin{aligned}
& \widehat{H}_{s i, 12}[k]=\frac{\widetilde{\alpha}^{*} H_{s i, a}[k]-\widetilde{\beta} T^{\prime}[k] \cdot H_{s i, b}^{*}\left[N_{n z}-k+1\right]}{|\widetilde{\alpha}|^{2}-|\widetilde{\beta}|^{2}} \\
& \widehat{H}_{s i, 21}[k]=\frac{\widetilde{\alpha}^{*} H_{s i, b}[k]-\widetilde{\beta} T^{\prime}[k] \cdot H_{s i, a}^{*}\left[N_{n z}-k+1\right]}{|\widetilde{\alpha}|^{2}-|\widetilde{\beta}|^{2}}
\end{aligned}
$$

The more accurate the estimation of $\widetilde{\alpha}$ and $\widetilde{\beta}$, the more closely the virtual self-interference channels $\widehat{H}_{s i, 12}[k]$ and $\widehat{H}_{s i, 21}[k]$ are to the self-interference channel $\widetilde{H}_{s i, 12}[k]$ and $\widetilde{H}_{s i, 21}[k]$. Therefore, the I/Q imbalance parameters $(\widetilde{\alpha}, \widetilde{\beta})$ that make the virtual self-interference channel $\widehat{H}_{s i, 1}[k]$ and $\widehat{H}_{s i, 2}[k]$ as flat as possible like the self-interference channels do are what we want to obtain. The mean square error (MSE) of the consecutive subcarrier channels is defined as the criteria to measure how flat the virtual self-interference channel is as follows

$$
\begin{aligned}
& M S E_{1}=\sum_{k}\left|\widehat{H}_{s i, 12}[k+1]-\widehat{H}_{s i, 12}[k]\right|^{2} \\
& M S E_{2}=\sum_{k}\left|\widehat{H}_{s i, 21}[k+1]-\widehat{H}_{s i, 21}[k]\right|^{2}
\end{aligned}
$$

For the symmetric analysis, the case $M S E_{1}$ is studied first. Then, according to (32), we 
obtain

$$
\begin{aligned}
& \left|\widehat{H}_{s i, 12}[k+1]-\widehat{H}_{s i, 12}[k]\right|^{2} \\
& =\frac{\left|\widetilde{\alpha}^{*} H_{s i, a}[k+1]-\widetilde{\beta} T^{\prime}[k+1] \cdot H_{s i, b}^{*}\left[N_{n z}-k\right]-\widetilde{\alpha}^{*} H_{s i, a}[k]+\widetilde{\beta} T^{\prime}[k] \cdot H_{s i, b}^{*}\left[N_{n z}-k+1\right]\right|^{2}}{\left(|\widetilde{\alpha}|^{2}-|\widetilde{\beta}|^{2}\right)^{2}} \\
& =\frac{\left|\widetilde{\alpha}^{*}\left(H_{s i, a}[k+1]-H_{s i, a}[k]\right)-\widetilde{\beta}\left(T^{\prime}[k+1] \cdot H_{s i, b}^{*}\left[N_{n z}-k\right]-T^{\prime}[k] \cdot H_{s i, b}^{*}\left[N_{n z}-k+1\right]\right)\right|^{2}}{\left(|\widetilde{\alpha}|^{2}-|\widetilde{\beta}|^{2}\right)^{2}}
\end{aligned}
$$

In order to simplify the expression (35), we define

$$
\begin{aligned}
\Delta H_{s i, a}[k] & =H_{s i, a}[k+1]-H_{s i, a}[k] \\
\Delta H_{s i, b}^{\prime}[k] & =T^{\prime}[k+1] \cdot H_{s i, b}^{*}\left[N_{n z}-k\right]-T^{\prime}[k] \cdot H_{s i, b}^{*}\left[N_{n z}-k+1\right]
\end{aligned}
$$

After plugging (36) and (37) into (35), it can be rewritten as

$$
\left|\widehat{H}_{s i, 12}[k+1]-\widehat{H}_{s i, 12}[k]\right|^{2}=\frac{\left|\widetilde{\alpha}^{*} \cdot \Delta H_{s i, a}[k]-\widetilde{\beta} \cdot \Delta H_{s i, b}^{\prime}[k]\right|^{2}}{\left(|\widetilde{\alpha}|^{2}-|\widetilde{\beta}|^{2}\right)^{2}}
$$

Since the self-interference channel is a flat fading channel, the adjacent subcarrier channel should be very close to each other. However, the I/Q imbalance parameters $(\widetilde{\alpha}$ and $\widetilde{\beta})$ increase the fluctuation of the flat fading self-interference channel $\widehat{H}_{s i, 12}[k]$ and $\widehat{H}_{s i, 21}[k]$. Then, the parameters $(\widetilde{\alpha}$ and $\widetilde{\beta})$ which make the $\left|\widehat{H}_{s i, 12}[k+1]-\widehat{H}_{s i, 12}[k]\right|$ as small as possible are the values we want to obtain. Therefore, the optimization problem can be expressed as

$$
\begin{aligned}
\left(\widetilde{\alpha}_{o, 1}, \widetilde{\beta}_{o, 1}\right) & =\arg \min _{\widetilde{\alpha} \in \mathcal{C}, \widetilde{\beta} \in \mathcal{C}}\left\{\sum_{k}\left\|\widehat{H}_{s i, 12}[k+1]-\widehat{H}_{s i, 12}[k]\right\|^{2}\right\} \\
& =\arg \min _{\widetilde{\alpha} \in \mathcal{C}, \widetilde{\beta} \in \mathcal{C}}\left\{\frac{\sum_{k}\left\|\widetilde{\alpha}^{*} \cdot \Delta H_{s i, a}[k]-\widetilde{\beta} \cdot \Delta H_{s i, b}^{\prime}[k]\right\|^{2}}{\left(|\widetilde{\alpha}|^{2}-|\widetilde{\beta}|^{2}\right)^{2}}\right\}
\end{aligned}
$$

where $\mathcal{C}$ denotes the set of complex number.

In order to get the optimal estimated values $\left(\widetilde{\alpha}_{o, 1}, \widetilde{\beta}_{o, 1}\right)$, taking the partial derivative of the $M S E_{1}$ with respect to $\widetilde{\beta}$ as

$$
\begin{aligned}
\frac{\partial M S E_{1}}{\partial \widetilde{\beta}} & =\frac{\partial\left\{\sum_{k}\left\|\widehat{H}_{s i, 12}[k+1]-\widehat{H}_{s i, 12}[k]\right\|^{2}\right\}}{\partial \widetilde{\beta}} \\
& =\sum_{k} \frac{\partial\left\{\frac{\left\|\widetilde{\alpha}^{*} \cdot \Delta H_{s i, a}[k]-\widetilde{\beta} \cdot \Delta H_{s i, b}^{\prime}[k]\right\|^{2}}{\left(|\widetilde{\alpha}|^{2}-|\widetilde{\beta}|^{2}\right)^{2}}\right\}}{\partial \widetilde{\beta}}
\end{aligned}
$$


Since $\left(|\widetilde{\alpha}|^{2}-|\widetilde{\beta}|^{2}\right)^{2} \approx 1$, remains valid for high gain imbalance $(\Delta A=0.2)$ and phase imbalance $(\Delta \phi=10)$, then (40) can be simplified as

$$
\frac{\partial M S E_{1}}{\partial \widetilde{\beta}} \approx \sum_{k} \frac{\partial\left\|\widetilde{\alpha}^{*} \cdot \Delta H_{s i, a}[k]-\widetilde{\beta} \cdot \Delta H_{s i, b}^{\prime}[k]\right\|^{2}}{\partial \widetilde{\beta}}=\sum_{k} \frac{\partial z_{k}}{\partial \widetilde{\beta}}
$$

where $z_{k} \triangleq\left\|\widetilde{\alpha}^{*} \cdot \Delta H_{s i, a}[k]-\widetilde{\beta} \cdot \Delta H_{s i, b}^{\prime}[k]\right\|^{2}$ which is function of $\widetilde{\beta}=\Re(\widetilde{\beta})+\jmath \Im(\widetilde{\beta})$.

and (30) show that the complex value $\widetilde{\beta}$ is the key factor that makes the originally flat self-interference channel fluctuate more. Therefore, the problem of calculating the optimal $\widetilde{\beta}$ in (41) becomes solving the Cauchy-Riemann equations as following

$$
\begin{gathered}
\sum_{k} \frac{\partial \Re\left(z_{k}\right)}{\partial \Re(\widetilde{\beta})}=\sum_{k} \frac{\partial \Im\left(z_{k}\right)}{\partial \Im(\widetilde{\beta})} \\
\sum_{k}-\frac{\partial \Re\left(z_{k}\right)}{\partial \Im(\widetilde{\beta})}=\sum_{k} \frac{\partial \Im\left(z_{k}\right)}{\partial \Re(\widetilde{\beta})}
\end{gathered}
$$

As it can be seen, the definition $z_{k}$ is function of $\widetilde{\beta}$, which can be expressed as

$$
\begin{aligned}
z_{k}= & |\widetilde{\alpha}|^{2}\left|\Delta H_{s i, a}[k]\right|^{2}+|\widetilde{\beta}|^{2}\left|\Delta H_{s i, b}^{\prime}[k]\right|^{2}-\widetilde{\alpha} \widetilde{\beta} \cdot \Delta H_{s i, a}^{*}[k] \cdot \Delta H_{s i, b}^{\prime}[k] \\
& -\widetilde{\alpha}^{*} \widetilde{\beta}^{*} \cdot \Delta H_{s i, a}[k] \cdot \Delta H_{s i, b}^{\prime *}[k]
\end{aligned}
$$

Then, the real and image part of $z_{k}$ can be obtained as

$$
\begin{aligned}
\Re\left(z_{k}\right)= & |\widetilde{\alpha}|^{2}\left|\Delta H_{s i, a}[k]\right|^{2}+\left(\Re(\widetilde{\beta})^{2}+\Im(\widetilde{\beta})^{2}\right)\left|\Delta H_{s i, b}^{\prime}[k]\right|^{2} \\
& -2 \Re\left(\widetilde{\alpha} \cdot \Delta H_{s i, a}^{*}[k] \cdot \Delta H_{s i, b}^{\prime}[k]\right) \Re(\widetilde{\beta})+2 \Im\left(\widetilde{\alpha} \cdot \Delta H_{s i, a}^{*}[k] \cdot \Delta H_{s i, b}^{\prime}[k]\right) \Im(\widetilde{\beta}) \\
\Im\left(z_{k}\right)= & 0
\end{aligned}
$$

According to (45) and (46), (42) and (43) can be rewritten as

$$
\begin{gathered}
\sum_{k} \frac{\partial \Re\left(z_{k}\right)}{\partial \Re(\widetilde{\beta})}=0 \\
\sum_{k}-\frac{\partial \Re\left(z_{k}\right)}{\partial \Im(\widetilde{\beta})}=0
\end{gathered}
$$

Based on (47) and (48), the real and image part of the optimal estimated $\widetilde{\beta}$ can be obtained as

$$
\begin{aligned}
\Re\left(\widetilde{\beta}_{o, 1}\right) & =\frac{\sum_{k} \Re\left(\widetilde{\alpha}_{o, 1} \cdot \Delta H_{s i, a}^{*}[k] \cdot \Delta H_{s i, b}^{\prime}[k]\right)}{\sum_{k}\left|\Delta H_{s i, b}^{\prime}[k]\right|^{2}} \\
\Im\left(\widetilde{\beta}_{o, 1}\right) & =\frac{-\sum_{k} \Im\left(\widetilde{\alpha}_{o, 1} \cdot \Delta H_{s i, a}^{*}[k] \cdot \Delta H_{s i, b}^{\prime}[k]\right)}{\sum_{k}\left|\Delta H_{s i, b}^{\prime}[k]\right|^{2}}
\end{aligned}
$$


Therefore, the optimal value $\widetilde{\beta}_{o, 1}$ of the estimated $\widetilde{\beta}$ is expressed as

$$
\begin{aligned}
\widetilde{\beta}_{o, 1} & =\Re\left(\widetilde{\beta}_{o, 1}\right)+\jmath \Im\left(\widetilde{\beta}_{o, 1}\right) \\
& =\frac{\sum_{k} \alpha^{*} \cdot \Delta H_{s i, a}[k] \cdot \Delta H_{s i, b}^{\prime *}[k]}{\sum_{k}\left|\Delta H_{s i, b}^{\prime}[k]\right|^{2}} \\
& =\widetilde{\alpha}_{0,1}^{*} \widetilde{\beta}_{1}
\end{aligned}
$$

Here, we define $\widetilde{\beta}_{1}$

$$
\begin{aligned}
\widetilde{\beta}_{1} & =\frac{\sum_{k} \Delta H_{s i, a}[k] \cdot \Delta H_{s i, b}^{\prime *}[k]}{\sum_{k}\left|\Delta H_{s i, b}^{\prime}[k]\right|^{2}} \\
& =\frac{\sum_{k}\left(H_{s i, a}[k+1]-H_{s i, a}[k]\right)\left(T^{\prime}[k+1] \cdot H_{s i, b}\left[N_{n z}-k\right]-T^{\prime}[k] \cdot H_{s i, b}\left[N_{n z}-k+1\right]\right)}{\sum_{k}\left|T^{\prime}[k+1] \cdot H_{s i, b}\left[N_{n z}-k\right]-T^{\prime}[k] \cdot H_{s i, b}\left[N_{n z}-k+1\right]\right|^{2}}
\end{aligned}
$$

That means $\widetilde{\beta}_{1}$ can be estimated by using the estimated self-interference channel coefficients $H_{s i, a}[k]$ and $H_{s i, b}[k]$ which are corrupted by the I/Q imbalance parameters $(\alpha$ and $\beta$ ). After getting the estimated $\widetilde{\beta}_{1}$, the optimal estimated value $\widetilde{\beta}_{o, 1}$ can be obtained by

$$
\widetilde{\beta}_{o, 1}=\widetilde{\alpha}_{o, 1}^{*} \cdot \widetilde{\beta}_{1}
$$

According to the characterization of $\widetilde{\alpha}_{o, 1}$ and $\widetilde{\beta}_{o, 1}$ as in (22) and (23), we can get

$$
\widetilde{\alpha}_{o, 1}+\widetilde{\beta}_{o, 1}^{*}=1
$$

Then, the optimal estimated I/Q imbalance parameters $\widetilde{\alpha}_{o}$ and $\widetilde{\beta}_{o}$ can be solved as

$$
\begin{aligned}
& \widetilde{\alpha}_{o, 1}=\frac{1}{1+\widetilde{\beta}} \\
& \widetilde{\beta}_{o, 1}=1-\widetilde{\alpha}_{o, 1}^{*}
\end{aligned}
$$

The same method can be used to obtain another pair of optimal I/Q imbalance parameters $\widetilde{\alpha}_{o, 2}$ and $\widetilde{\beta}_{o, 2}$ by solving the optimization problem

$$
\left(\widetilde{\alpha}_{o, 2}, \widetilde{\beta}_{o, 2}\right)=\arg \min _{\widetilde{\alpha} \in \mathcal{C}, \widetilde{\beta} \in \mathcal{C}}\left\{\sum_{k}\left\|\widehat{H}_{s i, 21}[k+1]-\widehat{H}_{s i, 21}[k]\right\|^{2}\right\}
$$

Then, the optimal estimated value $\widetilde{\alpha}_{o}$ and $\widetilde{\beta}_{o}$ which are employed for compensating the receiver I/Q imbalance are

$$
\begin{aligned}
\alpha_{o} & =\frac{\widetilde{\alpha}_{o, 1}+\widetilde{\alpha}_{o, 2}}{2} \\
\beta_{o} & =\frac{\widetilde{\beta}_{o, 1}+\widetilde{\beta}_{o, 2}}{2}
\end{aligned}
$$




\section{B) I/Q Imbalance Mitigation}

With the estimated I/Q imbalance parameters $\alpha_{o}$ and $\beta_{o}$ available, the I/Q imbalance existing in the FDDB RF front-end can be compensated and the two different symbol sequences carried by the two different carrier frequencies can be separated by solving (20) and (21) as

$$
\begin{gathered}
\widetilde{s}_{1}=\frac{\alpha_{o}^{*} s_{a}-\beta_{o} s_{b}^{*}}{\left|\alpha_{o}\right|^{2}-\left|\beta_{o}\right|^{2}} \\
\widetilde{s}_{2}=\frac{\alpha_{o}^{*} s_{b}-\beta_{o} s_{a}^{*}}{\left|\alpha_{o}\right|^{2}-\left|\beta_{o}\right|^{2}}
\end{gathered}
$$

The proposed I/Q imbalance estimator and compensation for the FDDB OFDM radio receiver is as shown in Fig. 8. The two baseband symbol sequences $\widetilde{s}_{1}$ and $\widetilde{s}_{2}$ after I/Q imbalance compensation will be processed by two baseband WLAN receivers as previously shown in Fig. 2. After that, the two data sequences from the distant radio node can be recovered.

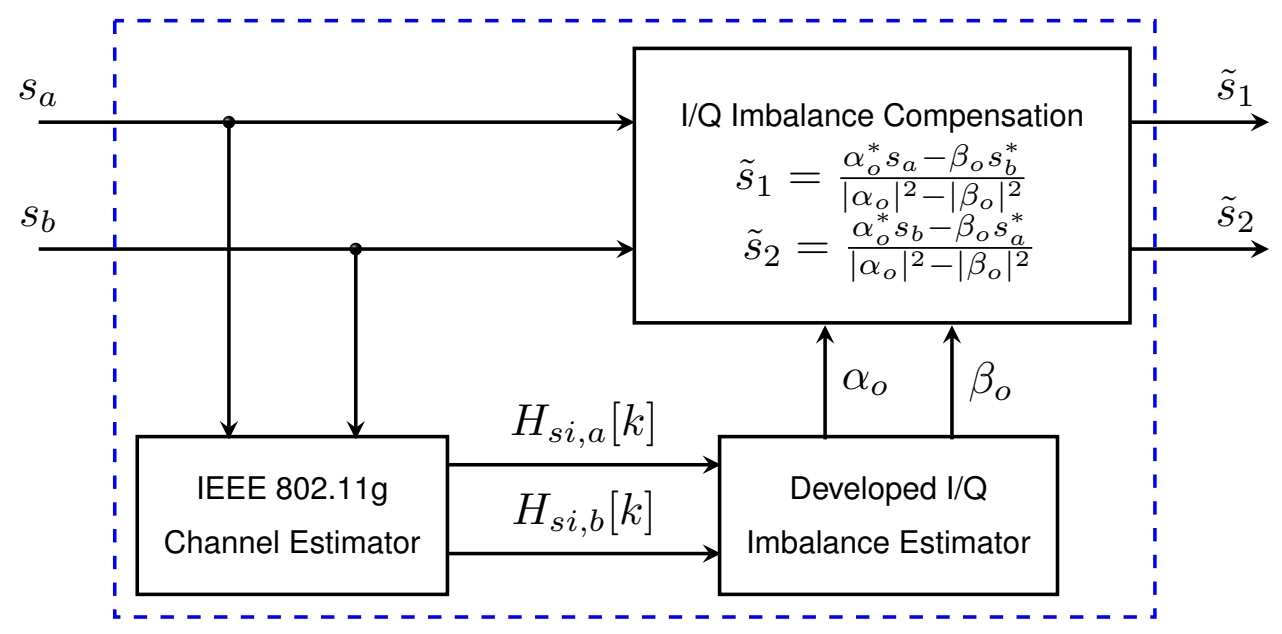

Figure 8: The compensation block for I/Q imbalance.

\section{C) Algorithm Procedure}

The procedure of the I/Q imbalance estimation and compensation is described in Algorithm 1. The received two baseband long training signals $\left(s_{l t s, a}, s_{l t s, b}\right)$ are used to obtain the channel estimates $\left(H_{s i, a}[k], H_{s i, b}[k]\right)$ by least square (LS) algorithm. Then, the optimal estimates of the I/Q imbalance parameters $\left(\alpha_{o}, \beta_{o}\right)$ are calculated by cooperatively utilize $\left(H_{s i, a}[k], H_{s i, b}[k]\right)$. Finally, $\left(\alpha_{o}, \beta_{o}\right)$ are used to eliminate the image part in $\left(s_{a}, s_{b}\right)$ and then the cleaned signal $\left(\tilde{s}_{1}, \tilde{s}_{2}\right)$ can be obtained.

\section{Numerical Results and Discussion}

In order to construct a link level simulation platform to demonstrate the proposed FDDB OFDM radio and validate the effective I/Q imbalance compensation algorithm, we model 


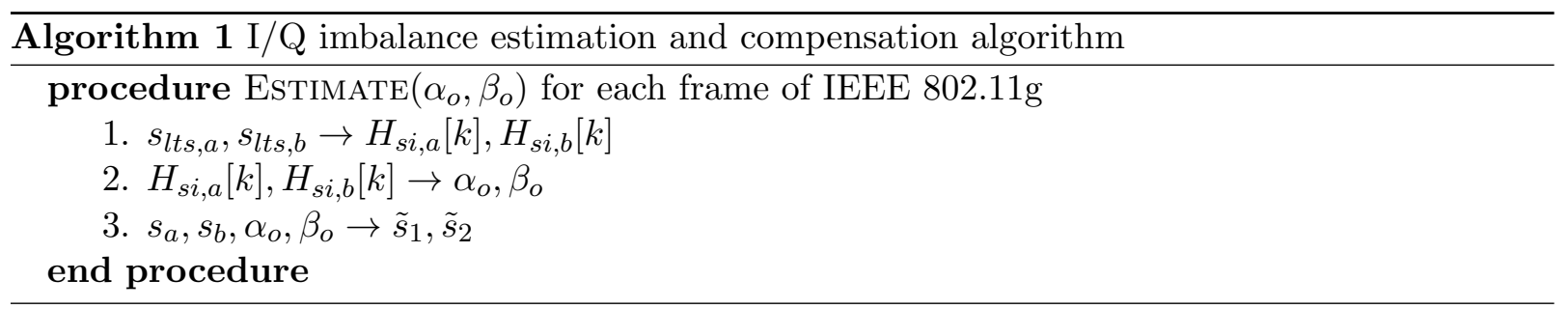

the RF front-end and most of the baseband systems on ADS (Advanced Design System, Agilent Technologies) software and part of digital signal processing and algorithm implementation on Matlab. Specifically, the digital estimation and compensation of the I/Q imbalance are carried out on Matlab.

In this section, the radio architecture proposed in this paper is demonstrated and validated by ADS-Matlab co-simulations. Additionally, the performance of FDDB OFDM radio transceiver with or without I/Q imbalance are presented and analyzed. The performance of the proposed radio transceiver with I/Q imbalance is significantly reduced by the mutual signal leakages. The loss of the performance caused by the I/Q imbalance can be compensated by the developed I/Q compensation method.

A typical OFDM based 36Mbps $802.11 \mathrm{~g}$ physical layer is employed to construct a FDDB OFDM radio link. The parameters used in the simulation are as shown in Tab. 1 according to the IEEE $802.11 \mathrm{~g}$ WLAN standard.

Table 1: System Parameters

\begin{tabular}{cc}
\hline \hline Parameter & Value \\
\hline Bandwidth & $20 \mathrm{MHz}$ \\
Total number of subcarriers & 52 \\
Number of data subcarriers & 48 \\
Number of pilot subcarriers & 4 \\
Bit rate & $36 \mathrm{Mbps}$ \\
IFFT/FFT period & $3.2 \mathrm{us}$ \\
GI duration & $0.8 \mathrm{us}$ \\
\hline
\end{tabular}

Generally, the signal emitted from each of the antennas has the same power level and the signals of interest with different carrier frequencies go through almost the same propagation loss $L$ and the self-interference signals also experience almost the same propagation loss $L_{s i}$, based on which we make an assumption that each receiver has the same signal-to-noise ratio (SNR) and the same self-interference-to-noise ratio (INR).

\section{A) Numerical Results}

The Bit Error Rate (BER) versus $E b / N o$ for the proposed radio link are calculated by ADS-Matlab co-simulation and as shown in Fig. 9-12. In all these figures, "FDDB WiFi-1" and "FDDB WiFi-2" refer to the FDDB IEEE 802.11g signals with carrier frequency $f_{1}=2.4 \mathrm{GHz}$ and $f_{2}=2.2 \mathrm{GHz}$ respectively; "Ideal IQ" refers to the radio receiver without I/Q imbalance; "Conventional WiFi Link" refers to the standard IEEE 802.11g wireless link which is used as the reference; "with IQ Imb./No Comp." refers to 


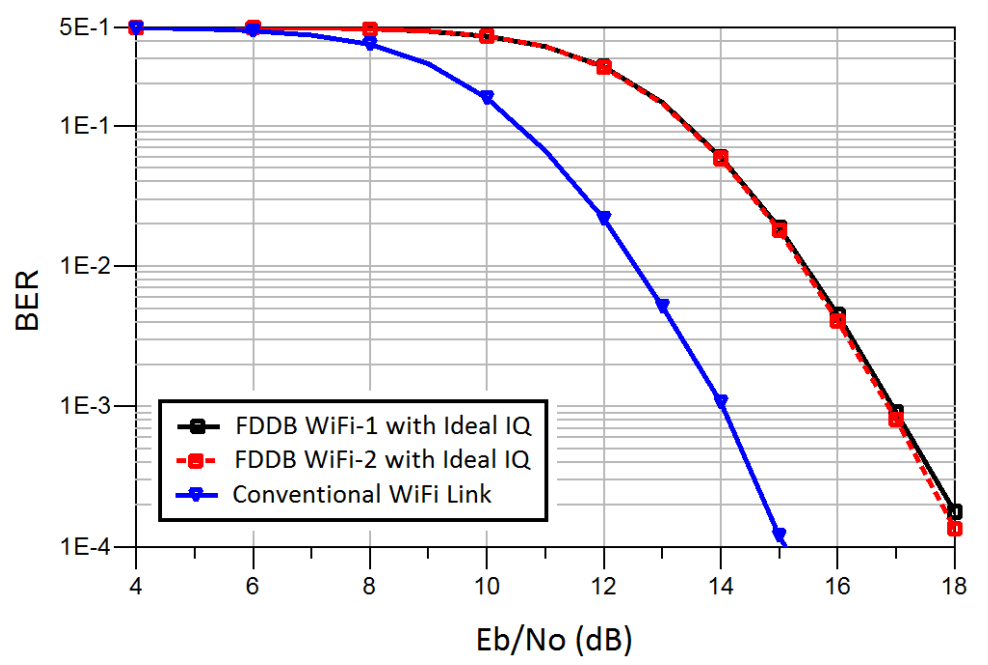

Figure 9: The BER of Full-Duplex Dual-Band 802.11g wireless system, with ideal I/Q.

the proposed radio receiver with I/Q imbalance but without I/Q imbalance compensation; "with I/Q Imb./Comp." refers to the proposed radio receiver with I/Q imbalance and with the I/Q imbalance compensation algorithm.

\section{1) FDDB OFDM Radio Transceiver without I/Q Imbalance}

As shown in Fig. 9, the BER curve of the FDDB WiFi-1 overlap with that of the FDDB WiFi-2. This is because these two signals have the same signal-to-interference-and-noise ratio (SINR) which relies on a reasonable assumption that each signal of interest has the same SNR and INR. However, there is around $3 \mathrm{~dB}$ performance loss for the FDDB WiFi compared to the conventional WiFi link. This can be explained by the noise-level residual self-interference induced by the Full-Duplex OFDM radio as presented in section II.

\section{2) FDDB OFDM Radio Transceiver with I/Q Imbalance}

The I/Q imbalance in the FDDB RF front-end causes undesired signal leakage as studied in section III. The leaked image signal superimposed on the signal of interest degrades the performance of reception.

As it can be seen in Fig. 10, the high level I/Q imbalance $\left(\Delta A=0.2, \Delta \phi=10^{\circ}\right)$ seriously degrades the proposed FDDB WiFi system. However, the proposed system performs very close to the ideal I/Q scenario after using the I/Q imbalance mitigation algorithm as presented in section III. Specifically, there is about $1 \mathrm{~dB}$ residual leaked image signal caused by the I/Q imbalance estimation error when $B E R=10^{-4}$.

As shown in Fig. 11, even with a medial level I/Q imbalance $\left(\Delta A=0.1, \Delta \phi=5^{\circ}\right)$, there is around $5.5 \mathrm{~dB}$ gap induced by the leaked image signal when $B E R=10^{-4}$. While after the I/Q imbalance compensation, the performance loss is negligible.

In order to show that the developed compensation method can also be applied to combat the low I/Q imbalance, we have simulated the scenario $\left(\Delta A=0.05, \Delta \phi=1^{\circ}\right)$ as shown in Fig. 12. Note that the BER curve of FDDB WiFi system with low I/Q imbalance is very close to that of the ideal case. This is because the low I/Q imbalance causes low leaked 


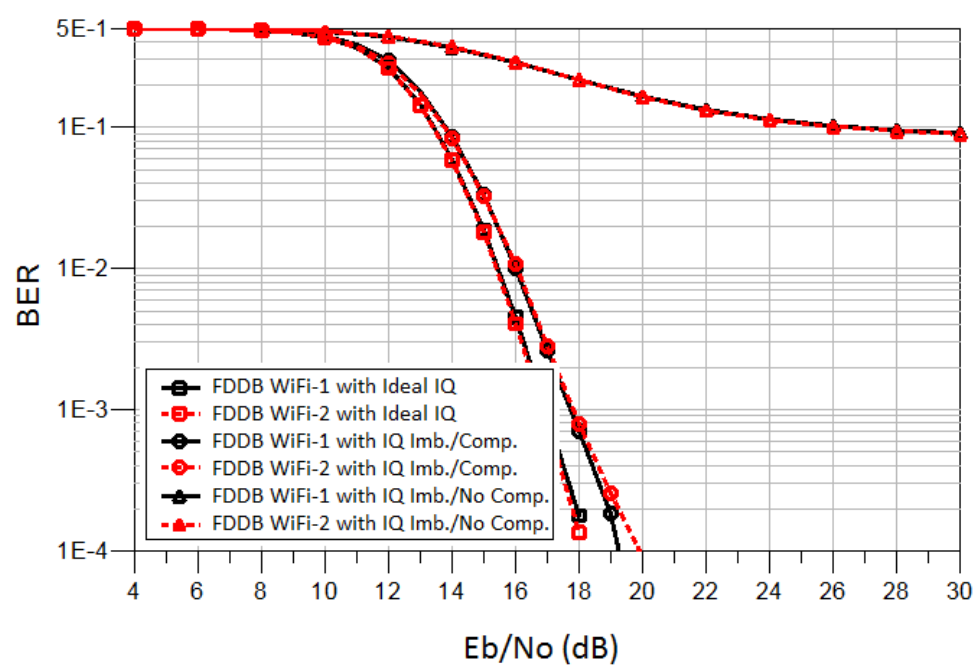

Figure 10: The BER of Full-Duplex Dual-Band 802.11g wireless system, with I/Q imbalance ( $\Delta A=0.2, \Delta \phi=10^{\circ}$.

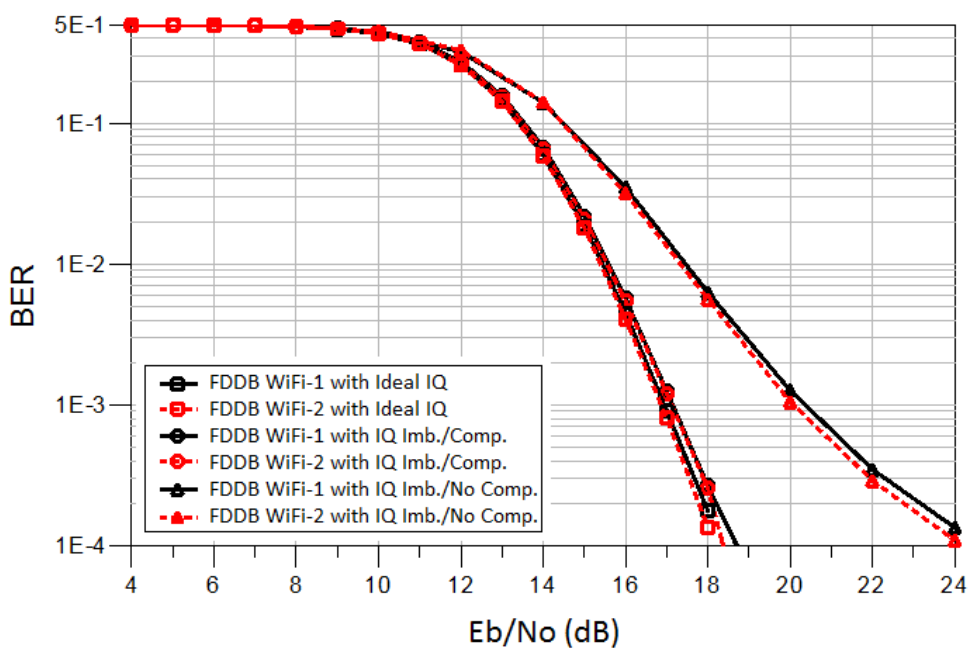

Figure 11: The BER of Full-Duplex Dual-Band 802.11g wireless system, with I/Q imbalance $\left(\Delta A=0.1, \Delta \phi=5^{\circ}\right)$. 


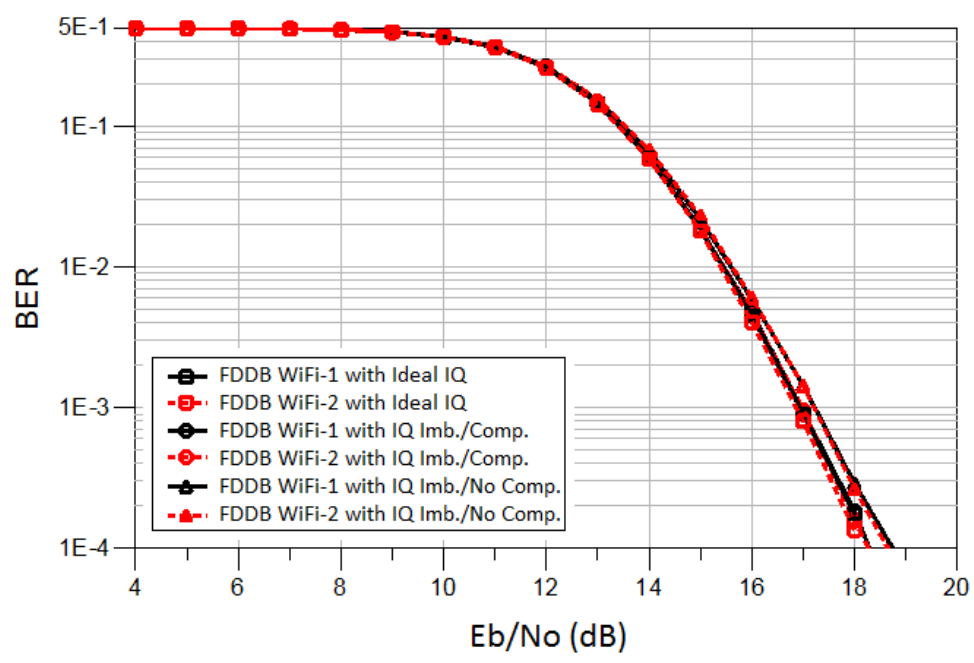

Figure 12: The BER of Full-Duplex Dual-Band 802.11g wireless system, with I/Q imbalance $\left(\Delta A=0.05, \Delta \phi=1^{o}\right)$.

image signal to the signal of interest. It can also be shown that the BER curve of the $\mathrm{I} / \mathrm{Q}$ imbalance corrupted FDDB WiFi system with compensation scheme almost overlap with the ideal scenario.

Additionally, the Fig. 9 shows that the proposed FDDB OFDM radio transceiver can simultaneously receive two different RF signals with carrier frequency $f_{1}$ and $f_{2}$ correctly while transmit another two different RF signals. Besides, comparing the BER performance of the proposed FDDB OFDM radio transceiver without or with different level of I/Q mismatch shows that the I/Q imbalance in the FDDB RF front-end indeed affect the performance of the receiver. However, the developed I/Q imbalance combating method can compensate the RF impaired front-end and make it very close to the ideal scenario.

\section{B) Discussion}

In this paper, the assumption that the two signals of interest carried by the frequency $f_{1}$ and $f_{2}$ arrive at the FDDB RF front-end with equal power level is made, so the two WiFi signals operating on two separate spectrum channel have the same reception performance even with I/Q imbalance. However, this assumption does not hold all the time. For example, when the radio terminal receives two different $R F$ signals transmitted from different radio nodes, these two signals should have unequal power levels even if they are transmitted with the same power level, because they experience different fading and propagation loss. In this case, the signal with low power level will be significantly degraded by the undesired signal leakage induced by the I/Q imbalance. In this scenario, the I/Q imbalance mitigation is much more important to make sure that the weak signal can also be recovered correctly.

The FDDB radio could be used in a lot of applications, but we can particularly emphasize one: Cognitive Radio. In fact, Cognitive Radios (CR) can be defined as intelligent radio systems which have the both abilities to sense the surrounding spectrum environment and flexibly adapt its transceiver parameters accordingly to optimize the radio link while 
coexisting with primary users [29]. It could greatly improve the efficiency of spectrum utilization. However, cognitive radios put high requirements on the RF front-end which is required to be highly flexible and reconfigurable and to realize wideband and agile transceiver. For example, the secondary user in cognitive radio systems can only use the spectrum channel unused or released by the primary user. Once the primary user starts to utilize this spectrum channel again, the secondary user should sense it and release this spectrum channel as soon as possible, and handover to another spectrum channel immediately. Therefore, the cognitive radio transceiver should be capable of spectrum channel sensing and provide candidate spectrum channel for handover. The proposed FDDB radio could meet these requirements. Full-Duplex wireless is defined as simultaneous wireless transmit and receive in the same frequency band $[8,12]$, so the Full-Duplex enables the radio terminals to sense spectrum channel while it is transmitting and receiving [30]. Dual-Band radio can simultaneously receive two different carrier frequency signals via using one RF front-end with double I/Q structure [4], so it can realize multiband radio with a relative simple RF front-end. Besides, OFDM's capabilities of spectrum sensing and shaping and its flexibility and adaptivity render it probably the best physical technique for cognitive radio systems [5]. Therefore, FDDB OFDM radio transceiver is proposed as a candidate radio transceiver for the cognitive radios. Besides, Full-Duplex OFDM radio has high requirements on the time and frequency synchronization to facilitate the active self-interference cancellation. While in the practical system design, the requirement of perfect time and frequency synchronization can not be always met. Therefore, more advanced and robust time and frequency synchronization algorithms $[31,32]$ are needed to eliminate the time and frequency synchronization errors.

\section{Conclusion}

In this article, a flexible radio: FDDB radio is presented. In order to study and demonstrate the potential of this flexible radio, the system level simulation framework is built by combining the FDDB radio and WiFi OFDM PHY and make it operate on two separate spectrum fragments. The self-interference including the inner self-interference caused by the one-antenna Full-Duplex and the internal self-interference induced by the two-antenna Full-Duplex have been suppressed or canceled to noise level by creatively using the available self-interference cancellation methods. The ADS-Matlab co-simulation results show that the studied flexible radio transceiver based on the proposed flexible radio could simultaneously receive two different types of signals with a relative simple $R F$ front-end at the cost of $3 \mathrm{~dB}$ loss in BER, which is tolerable when the system capacity has almost 4 times enhancement. However, the noticeable gain in the radio link capacity disappears when I/Q imbalance exists in the FDDB RF front-end. In order to mitigate the I/Q imbalance, an advanced and suitable I/Q imbalance estimation and compensation method dedicated to the FDDB OFDM radio is developed based on the frequency-flat-fading characteristic of the self-interference channel.

The self-interference can be canceled to the noise level and the viable system performance can be obtained based on the assumption that the over-the-air self-interference channel is one-path one-delay channel relying on the fact that the power of the line-of-sight path dominates the self-interference channel. However, in practical consideration, the 
over-the-air self-interference channel should be a multi-path wireless channel with higher Rician $K$ factor which denotes the relative strength of the direct and scattered components of the received signals. Therefore, as a perspective of this work, designing or developing a more robust self-interference cancellation algorithm is an important future work.

Besides, the phase noise in the local oscillator would be another important factor affecting the FDDB OFDM radio transceivers. Especially, it will affect the self-interference cancellation in two-antenna Full-Duplex OFDM wireless. Hence, studying and eliminating the impact of phase noise on the proposed radio system is also our future work. Furthermore, it was mentioned that the FDDB OFDM radio could be a good radio transceiver candidate for the cognitive radios, because this radio could enable the radio terminal to carry out the spectrum channel sensing while working and provide the candidate spectrum channel for hand-over. However, this viable radio performance has not been demonstrated experimentally. Therefore, prototyping the proposed radio system on hardware and demonstrating the actual performance are also considered as future works.

\section{REFERENCES}

[1] Zhan, Z; Villemaud, G.; Hutu, F.; Gorce, J.-M.: Full-Duplex Dual-Band radio dedicated to flexible radio system, INRIA Research Report, RR-8558, Jul. 2014.

[2] Duarte, M; Sabharwal, A.: Full-Duplex wireless communication using off-the-shelf radios: Feasibility and first results, IEEE Asilomar Conference on Signals, Systems and Computers, Pacific Grove, CA, 2009.

[3] Jain, M.; Choi, J.; Kim, T.; Bharadia, D.; Srinivasan, K; Levis, P.; Katti, S.; Sinha, P.; Seth, S.: Practical real-time Full-Duplex wireless, ACM Mobicom, Las Vegas, Nevada, 2011.

[4] Burciu, I.; Villemaud, G.; Verdier, J.; Gautier, M.: Low power front-end architecture dedicated to the multistandard simultaneous reception, Cambridge Int. J. Microwave and Wireless Tech., vol. 2, no. 6, 504-514, 2010.

[5] Hahmoud, H.; Yucek, T.; Arslan, H.: OFDM for cognitive radio: merits and challenges, IEEE Wireless Communications, vol. 16, no. 2, 6-15, 2009.

[6] Khandani, A.K.: Methods for Spatial Multiplexing of Wireless Two-Way Channels, US Patent, 2010.

[7] Khandani, A.K.: Two-Way (True Full-Duplex) Wireless, 13th Canadian Workshop on Information Theory, 2013.

[8] Choi, J.; Jain, M.; Srinivasan, K; Levis, P.; Katti, S.: Achieving single channel, Full-Duplex wireless communications, ACM MobiCom, Chicago, Illinois, 2010.

[9] Aryafar, E.; Khojastpour, M.; Sundaresan, K; Rangarajan, S.; Chiang, M.: MIDU: Enabling MIMO Full-Duplex, ACM MobiCom, Istanbul, Turkey, 2012.

[10] Mohammad, A.; Sundaresan, K; Rangarajan, S.; Zhang, X.; Barghi, S.: The Case for Antenna Cancellation for Scalable Full-Duplex Wireless Communications, ACM Hotnets, Cambridge, MA, USA, 2011. 
[11] Everett, E.; Sahai, A.; Sabharwal, A.: Passive self-interference suppression for Full-Duplex infrastructure nodes, IEEE Transaction on Wireless Communications, vol. 13, no. 2, 680-694, 2014 .

[12] Sahai, A.; Patel, G.; Sabharwal, A.: Pushing the limits of Full-Duplex: Design and real-time implementation, Rice University, Technical Report TREE1104, 2011.

[13] Kaufman, B.; Lilleberg, J.; Aazhang, B.: Analog baseband cancellation for Full-Duplex: An experiment driven analysis, online: http://arxiv.org/pdf/1312.0522v1.pdf.

[14] Tarighat, H.; Bagheri, R.; Sayed, A.: Compensation schemes and performance analysis of IQ imbalance in OFDM receivers, IEEE Transaction on Signal Processing, vol. 53, no. 8, 3257-3268, 2005.

[15] Sohn, I.; Jeong, E.; Lee, Y.: Data-aided approach to I/Q mismatch and DC offset compensation in communication receivers, IEEE Communications Letters, vol. 6, no. 12, 547-549, 2002 .

[16] Windisch, M.; Fettweis, G.; Preamble design for an efficient I/Q compensation in OFDM direct-conversion receivers, 10th International OFDM workshop, 2005.

[17] Tubbax, J.; Come, B.; Perre, L.D.; Donnay, S.; Engels, H.; Man, H.; Moonen, M.: Compensation of IQ imbalance and phase noise in OFDM systems, IEEE Transaction on Wireless Communications, vol. 4, no. 3, 872-877, 2005.

[18] Yu, J.; Sun, M.; Hus, T.; Lee, C.: A novel technique for I/Q imbalance and CFO compensation in OFDM systems, IEEE International Symposium on Circuit and Systems, 2005.

[19] Windisch, M.; Fettweis, G.; Standard-independent I/Q imbalance compensation in OFDM direct-conversion receivers, 9th International OFDM workshop, 2004.

[20] Traverso, S.; Ariaudo, M.; Fijalkow, I.; Gautier, J.; Lereau, C.: Decision-directed channel estimation and high I/Q imbalance compensation in OFDM receivers, IEEE Transaction on Communications, vol. 57, no. 5, 1246-1249, 2009.

[21] Gil, G.; Kim, Y.; Lee, Y.: Non-data-aided approach to I/Q mismatch compensation in low-IF receivers, IEEE Transaction on Signal Processing, vol. 55, no. 7, 3360-3365, 2007.

[22] Windisch, M.; Fettweis, G.; Blind I/Q imbalance parameter estimation and compensatino in low-IF receivers, First International Symposium on Control, Communications and Signal Processing, 2004.

[23] Bharadia, D.; McMilin, E.; Katti, S.: Full Duplex radios, ACM SigCom, Hongkong, China, 2013.

[24] Hong, S.; McMilin, E.; Katti, S.: Picasso: flexible RF and spectrum slicing, ACM SigCom, Helsinki, Finland, 2012.

[25] Sabharwal, A.; Schniter, P.; Guo, D.; Bliss, D.-W.; Rangarajan, S.; Wichman, R.; In-Band Full-Duplex Wireless: Challenges and Opportunities, IEEE Journal on Selected Areas in Communications, vol. 32, no. 9, 1637-1652, 2014. 
[26] Duarte, M.; Dick C.; Sabharwal, A.: Experiment Driven characterization of Full-Duplex wireless communications, IEEE Transaction on Wireless Communications, vol. 11, no. 12, 505-514, 2012.

[27] Zhan, Z; Villemaud, G.; Gorce, J.-M.: Design and evaluation of a wideband Full-Duplex OFDM system based on AASIC, IEEE 24th Annual International Symposium on Personal, Indoor and Mobile Radio Communication, London, UK, 2014.

[28] Zhan, Z; Villemaud, G.; Gorce, J.-M.: Analysis and reduction of the impact of thermal noise on the Full-Duplex OFDM radio, IEEE Radio and Wireless symposium, Newport Beach, CA, 2014.

[29] Cabric, D; Mishra, S.; Brodersen, R.: Implementation issues in spectrum sensing for cognitive radios, IEEE Asilomar Conference on Signals, Systems and Computers, 2014.

[30] Choi, Y.; Hooman, S.: Simultaneous transmission and reception: Algorithm, design ans system level performance, IEEE Transaction on Wireless Communications, vol. 12, no. 12, 5992-6010, 2013.

[31] Schmidl, T. M.; Cox, D.: Robust Frequency and Timing Synchronization for OFDM, IEEE Transaction on Communications, vol. 45, no. 12, 1613-1621, 1997.

[32] Minn, H.; Bhargava, V. K.; Letaief, K. B.: A Robust Timing and Frequency Synchronization for OFDM Systems, IEEE Transaction on Wireless Communications, vol. 2, no. 4, 822-839, 2003.

Bibliographies (no more than 150 words each, accompanied by a 75 x 88 pixel photo)

Zhaowu Zhan received the M.Sc. degree in communications and information system from Xidian University, Xi'an, China, in 2011. Currently, he is working toward the Ph.D. degree at CITI laboratory of INSA Lyon, France. His main research interests are Full-Duplex OFDM radio, Dual-Band MIMO radio, and flexible radio transceiver design.

Guillaume Villemaud received the M.S. degree in EE from the University of Limoges, France, in 1999. From 1999 to 2002 he worked at IRCOM on compact integrated antennas and received his PhD degree in electronics in 2002. In 2013, he obtained his HDR (Qualification to supervise research) from INSA Lyon and Université Lyon 1. He developed multi-band hybrid arrays for CREAPE from 2002 to 2003 and then joined the CITI Laboratory, Lyon, France, in 2003. He is currently Associate Professor at INSA Lyon, Head of the International office of the EE Department. He is now Vice-Head of the CITI Lab. His research interests are antenna diversity, multiple antenna processing, $\mathrm{RF}$ architectures and system level simulation, coverage prediction, radio propagation and measurements. He is also responsible of the 'Flexible radio Front-ends' axis of INRIA SOCRATE. He has published more than 100 international technical papers and is a Senior Member of IEEE.

Florin Hutu received in 2007 a $\mathrm{PhD}$ in automatic control from University of Poitiers, France. Between 2008 and 2010 he was a postdoctoral fellow at Xlim Laboratory, France studying radio front ends architecture for beamforming. Since September 2010 he joined CITI laboratory, Lyon, France as an associated professor. His main research topic is the study of the radio front end architectures under energy constraints. 
Jean-Marie Gorce is a professor of Wireless Communications at the National Institute of Applied Sciences (INSA), Lyon, France. He joined INSA as an associate professor in 1999, becoming full Professor in 2008. Prof. Gorce received the Dipl. Ing. (M.Sc.) degree in electrical engineering (1993) and the PhD degree (1998) from INSA Lyon, working on radio-frequency ultrasound signals for echocardiography at CREATIS. After a postdoctoral year at Bracco Research, Geneva, Switzerland, he joined the Telecommunications Department at INSA Lyon in 1998. Since 2001 he has been a member of INRIA. Prof. Gorce is also a member of the joint lab between INRIA and Alcatel Lucent Bell Labs. His main research fields concern wireless communications focusing on realistic modeling, wireless system optimization and performance assessment considering both infrastructure-based and ad hoc networks. he has been involved as a leading scientist in several French and European projects related to wireless LAN planning, wireless sensor networks and body area networks. Prof. Gorce has published over 80 refereed journal and conference papers. He currently serves as an associate editor of Telecommunication Systems and Journal of Wireless Communications and Networking and has been TPC member of various international conferences.

\section{List of figures and tables}

Fig. 1. Block diagram of the Full-Duplex Dual-Band wireless radios.

Fig. 2. Full-Duplex Dual-Band OFDM radio transceiver.

Fig. 3. Architecture of Self-Interference Cancellation for in-band Full-Duplex wireless with two antennas.

Fig. 4. Frame structure for the Full-Duplex Dual-Band IEEE 802.11g.

Fig. 5. Architecture of Dual-Band RF front-end.

Fig. 6. Dual-Band RF front-end with I/Q imbalance.

Fig. 7. The estimated magnitude of each subcarrier channel of the over-the-air self-interference channel with or without I/Q Imbalance. When without I/Q imbalance, the estimated over-theair self-interference channel is almost a frequency flat fading channel, while this self-interference channel will be a frequency selective fading channel when I/Q imbalance $\left(\Delta A=0.2, \Delta \phi=10^{\circ}\right)$ exist in the Full-Duplex Dual-Band radio front-end.

Fig. 8. The compensation block for I/Q imbalance.

Fig. 9. The BER of Full-Duplex Dual-Band 802.11g wireless system, with ideal I/Q.

Fig. 10. The BER of Full-Duplex Dual-Band 802.11g wireless system, with I/Q imbalance ( $\left.\Delta A=0.2, \Delta \phi=10^{\circ}\right)$.

Fig. 11. The BER of Full-Duplex Dual-Band 802.11g wireless system, with I/Q imbalance ( $\Delta A=0.1, \Delta \phi=5^{\circ}$ ).

Fig. 12. The BER of Full-Duplex Dual-Band $802.11 \mathrm{~g}$ wireless system, with I/Q imbalance ( $\left.\Delta A=0.05, \Delta \phi=1^{\circ}\right)$.

Table 1. System Parameters. 